\title{
Regulation of intercellular biomolecule transfer-driven tumor angiogenesis and responses to anticancer therapies
}

\author{
Zhen Lu, ${ }^{1}$ Angelica Ortiz, ${ }^{1}$ loannis I. Verginadis, ${ }^{2}$ Amy R. Peck, ${ }^{3}$ Farima Zahedi, ${ }^{1}$ Christina Cho, ${ }^{1}$ Pengfei Yu, ${ }^{1}$ Rachel M. DeRita, ${ }^{1}$ \\ Hongru Zhang, ${ }^{1}$ Ryan Kubanoff, ${ }^{3}$ Yunguang Sun, ${ }^{4}$ Andrew T. Yaspan, ${ }^{4}$ Elise Krespan, ${ }^{5}$ Daniel P. Beiting, ${ }^{5}$ Enrico Radaelli, ${ }^{5}$ \\ Sandra W. Ryeom, ${ }^{6}$ J. Alan Diehl, ${ }^{7}$ Hallgeir Rui, ${ }^{4}$ Constantinos Koumenis, ${ }^{2}$ and Serge Y. Fuchs ${ }^{1}$
}

'Department of Biomedical Sciences, School of Veterinary Medicine, University of Pennsylvania, Philadelphia, Pennsylvania, USA. ²Department of Radiation Oncology, Perelman School of Medicine, University of Pennsylvania, Philadelphia, Pennsylvania, USA. ${ }^{3}$ Department of Chemistry, School of Arts and Sciences, University of Pennsylvania, Philadelphia, Pennsylvania, USA. ${ }^{4}$ Department of Pathology, Medical College of Wisconsin, Milwaukee, Wisconsin, USA. ${ }^{5}$ Department of Pathobiology, School of Veterinary Medicine, University of Pennsylvania, Philadelphia, Pennsylvania, USA. ${ }^{6}$ Department of Cancer Biology, Perelman School of Medicine, University of Pennsylvania, Philadelphia, Pennsylvania, USA. Department of Biochemistry, Case Western Reserve University School of Medicine, Cleveland, Ohio, USA.

\begin{abstract}
Intercellular biomolecule transfer (ICBT) between malignant and benign cells is a major driver of tumor growth, resistance to anticancer therapies, and therapy-triggered metastatic disease. Here we characterized cholesterol 25-hydroxylase (CH25H) as a key genetic suppressor of ICBT between malignant and endothelial cells (ECs) and of ICBT-driven angiopoietin-2dependent activation of ECs, stimulation of intratumoral angiogenesis, and tumor growth. Human $\mathrm{CH} 25 \mathrm{H}$ was downregulated in the ECs from patients with colorectal cancer and the low levels of stromal $\mathrm{CH} 25 \mathrm{H}$ were associated with a poor disease outcome. Knockout of endothelial $\mathrm{CH} 25 \mathrm{H}$ stimulated angiogenesis and tumor growth in mice. Pharmacologic inhibition of ICBT by reserpine compensated for $\mathrm{CH} 25 \mathrm{H}$ loss, elicited angiostatic effects (alone or combined with sunitinib), augmented the therapeutic effect of radio-/chemotherapy, and prevented metastatic disease induced by these regimens. We propose inhibiting ICBT to improve the overall efficacy of anticancer therapies and limit their prometastatic side effects.
\end{abstract}

\section{Introduction}

Within a multicellular organism, the horizontal exchange of biomolecules such as nucleic acids, polypeptides, lipids, and others between cells has emerged as an important mode of communication that encourages collective cell behavior (1). In a healthy organism, biomolecule exchange between diverse types of normal cells helps to maintain homeostatic balance and organize normal biologic processes (e.g., antigen presentation [refs. 2, 3] or normal angiogenesis [ref. 4]). However, in an organism that bears a malignant tumor, this intercellular biomolecule transfer (ICBT) from cancer cells to normal cells often stimulates tumor growth, progression, and metastasis (5-8).

ICBT can occur through a multitude of mechanisms, including uptake of extracellular vesicles and apoptotic bodies, cell fusion, trogocytosis, trans-endocytosis, cell junction and tunneling nanotube formation, and others (reviewed in ref. 9). In the context of tumorigenesis, ICBT mediated by tumor-derived extracellular vesicles (TEVs) has emerged as a pivotal driver of pathogenesis and outcome of oncologic diseases $(5,7)$. Biomolecules delivered by TEVs reprogram normal cells to contribute to many processes that promote tumor growth and progression, including modula-

Conflict of interest: The authors have declared that no conflict of interest exists. Copyright: (5) 2021, American Society for Clinical Investigation.

Submitted: September 11, 2020; Accepted: March 23, 2021; Published: May 17, 2021.

Reference information: / Clin Invest. 2021;131(10):e144225.

https://doi.org/10.1172/JCl144225. tion of metabolic activities, formation of a metastatic niche, suppression of immune responses, stimulation of angiogenesis, etc. (5, 7, 10-12).

Among other types of normal cells, endothelial cells (ECs) can become targets for ICBT. Intratumoral ECs in proximity to malignant cells were found to harbor genetic alterations similar to those found in the malignant cells, and ICBT between these cell types during cell fusion or efferocytosis has been proposed as a putative mechanism underlying this phenomenon (13). Subsequent studies confirmed transfer of tumoral DNA from malignant cells to ECs (14), demonstrated activation of ECs by TEVs (15), and established the paradigm supporting the important role of TEV-mediated ICBT in angiogenesis within tumor microenvironments (reviewed in refs. $5,8,16$ ).

Importantly, TEV-mediated ICBT often undermines the efficacy of anticancer therapies (17-19). Furthermore, radio- and chemotherapeutic agents increase production and/or release of TEVs by malignant cells; this increase is implicated in the iatrogenic metastatic disease arising from the treatment of primary tumors (20-25). Addressing these challenges requires a better understanding of genetic regulators of mechanisms underlying the protumorigenic ICBT. In addition, development of pharmacologic means to restrict ICBT should offer a novel approach to curtail tumor growth and progression and to improve the efficacy of existing anticancer therapies.

The biological barriers against ICBT are expected to preserve functional integrity of normal cells and restrict their cooperation 
with malignant cells. The type I interferon (IFN) pathway acts as one of those barriers that prevents generation of the prometastatic niche and pulmonary metastases (26-28). Cholesterol 25hydroxylase $(\mathrm{CH} 25 \mathrm{H})$, an enzyme that is induced by IFN (29-31), acts to catalyze the formation of 25-hydroxycholesterol $(25 \mathrm{HC})$. This oxysterol inhibits lipid membrane fusion (32) and, accordingly, uptake of TEVs (26). Uptake of TEVs by normal cells was also shown to be inhibited by the antihypertensive agent reserpine, which in turn could restore $\mathrm{CH} 25 \mathrm{H}$ expression otherwise downregulated by TEVs (26).

Here we characterize endothelial $\mathrm{CH} 25 \mathrm{H}$ as a key genetic suppressor of ICBT and of ICBT-driven activation of ECs, as well as of intratumoral angiogenesis and tumor growth. Our data also demonstrate that the suppressive effect of $\mathrm{CH} 25 \mathrm{H}$ on ICBT and ICBT-driven angiogenesis could be reenacted pharmacologically by administering reserpine, which, upon combination with different types of anticancer therapies, increases their efficacy and abolishes therapy-stimulated metastatic disease.

\section{Results}

$\mathrm{CH} 25 \mathrm{H}$ and reserpine inhibit ICBT between malignant cells and ECs. We previously reported that uptake of DiD dye-labeled TEVs is decreased in normal, wild-type (WT) splenocytes pretreated with reserpine or in splenocytes from knockin mice expressing a stabilized mutant of IFN receptor 1 (IFNAR1) (33). Importantly, the latter phenotype was lost upon ablation of Ch25h (26). Given that, in addition to TEVs, there are other mechanisms of biomolecule exchange such as uptake of apoptotic bodies, cell fusion, trans-endocytosis, etc. (9), we sought to determine the role of $\mathrm{CH} 25 \mathrm{H}$ expression in benign cells in regulating ICBT within solid tumors in vivo.

To this end, we grew subcutaneous tumors from B16F10-TdTomato melanoma cells in the flanks of WT or Ch25h/- mice that constitutively expressed green fluorescent protein (GFP) (Figure 1A). Tumors were harvested, dissociated, and analyzed for the numbers of $\mathrm{TdTomato}^{+} \mathrm{GFP}^{+}$double-positive cells among immune $\mathrm{CD} 45^{+}$immune and $\mathrm{CD}^{-} 5^{-}$nonimmune populations (Supplemental Figure 1, A and B; supplemental material available online with this article; https://doi.org/10.1172/JCI144225DS1). We found that tumors from the $C h 25 h^{-/-}$mice contained a greater number of $\mathrm{TdTomato}^{+} \mathrm{GFP}^{+}$cells in the $\mathrm{CD} 45^{-}$nonimmune stromal population (Figure 1B). Subsequent analysis of double-positive cells specifically in fibroblastic (PDGFR $\alpha^{+}$, Supplemental Figure 1C) and endothelial (CD31+, Supplemental Figure 1D) compartments revealed that ECs are the main target for the $\mathrm{CH} 25 \mathrm{H}$-regulated exchange of biomolecules with malignant cells (Figure 1C). The extent of ICBT in the ECs from B16F10-TdTomato tumors growing in $\mathrm{GFP}^{+}$mice was notably decreased by in vivo administration of reserpine (Figure 1D).

We next examined ICBT in vitro mediated by isolated TEVs characterized in Supplemental Figure 1, E-G. To safeguard against possible peculiarities of the intratumoral ECs in the GFP-expressing mice, we isolated ECs from the lungs of naive mice (Supplemental Figure 1, H and I) and treated them with TEVs isolated from B16F10 cells stably expressing GFP. A greater amount of Gfp mRNA was transferred into $\mathrm{CH} 25 \mathrm{H}$ deficient ECs compared with WT ECs; however, this phenotype was partially reversed upon treatment with reserpine (Figure 1E). Similar results were obtained when ICBT was assessed by transfer of DiD dye from TEVs into ECs (Supplemental Figure 1, $\mathrm{J}$ and $\mathrm{K}$ ). Collectively, these results suggest that $\mathrm{CH} 25 \mathrm{H}$ acts as a genetic suppressor of ICBT between malignant cells and ECs and characterize reserpine as a pharmacologic agent capable of inhibiting ICBT in vitro and in vivo.

Inactivation of stromal $\mathrm{CH} 25 \mathrm{H}$ promotes tumor growth and angiogenesis. $\mathrm{CH} 25 \mathrm{H}$ levels have been found to be decreased in leukocytes from tumor-bearing mice and melanoma patients compared with tumor-free control groups (26); however, the importance of $\mathrm{CH} 25 \mathrm{H}$ downregulation in normal cells for tumor growth is not completely understood. Intriguingly, during the course of experiments described in Figure 1A, we noticed that subcutaneous TdTomato-expressing B16F10 melanoma tumors grew faster in Ch25h/- compared with WT mice (Figure 2, A and B). Similar results were obtained with tumors formed by parental B16F10 cells (Supplemental Figure 2, A and B). This observation prompted us to examine the importance of $\mathrm{CH} 25 \mathrm{H}$ expression in the tumor microenvironment for growth of other types of cancer cells.

An accelerated growth of syngeneic tumors in $\mathrm{Ch} 25 \mathrm{~h}^{-/-}$(compared with WT) mice was observed for transplanted MH6499c4 pancreatic ductal adenocarcinoma (Figure 2C) and MC38 colon adenocarcinoma (Figure 2D and Supplemental Figure 2C). Furthermore, this phenotype was not limited to subcutaneous tumors, as $C h 25 h^{-/}$(compared with WT) mice exhibited a significantly faster growth of orthotopically transplanted MC38 colon tumors (Figure 2E) and TRAMP-C2-luciferase prostate neuroendocrine tumors (Figure 2F and Supplemental Figure 2D). Collectively, these data indicate that stromal $\mathrm{CH} 25 \mathrm{H}$ inhibits growth of different types of solid tumors.

Visual appearance of tumors growing in $C h 25 h^{-/-}$mice was suggestive of a greater extent of vascularization (as compared with WT; Figure 3A and Supplemental Figure 3A). This phenotype, as well as an increased ICBT involving ECs in $\mathrm{Ch} 25 \mathrm{~h}^{-/-}$mice (Figure 1), prompted us to examine the status of $\mathrm{CH} 25 \mathrm{H}$ and angiogenesis in the stromal compartment of human colorectal cancers (CRCs).

Analysis of tumors from an initial cohort of CRC patients (Cohort 1) revealed that levels of $\mathrm{CH} 25 \mathrm{H}$ were significantly downregulated in the stromal compartment of malignant colorectal tumors compared with stroma of benign adjacent colon tissue (Figure 3, B and C). Importantly, survival analysis in a cohort of CRC patients with available clinical follow-up data (Cohort 2) revealed that low levels of $\mathrm{CH} 25 \mathrm{H}$ within the tumor stroma were significantly associated with highly unfavorable prognosis (HR, 4.3; 95\% CI, 1.58-11.57; $P=0.004$; Figure 3D). Moreover, costaining of $\mathrm{CH} 25 \mathrm{H}$ and $\mathrm{CD} 31$ revealed that $\mathrm{CH} 25 \mathrm{H}$ levels specifically in the $\mathrm{CD} 31^{+}$ECs were notably downregulated in ECs within tumor stroma compared with ECs from healthy colon stroma, as shown first in an analysis of unmatched cases (Cohort 3, Figure 3E). These findings were further validated in an independent set of CRC cases with matched cancer stroma and nearby normal colon stroma (Cohort 4 , Figure $3 \mathrm{E}$ ). These results provide strong correlative support for the notion that downregulation of $\mathrm{CH} 25 \mathrm{H}$ occurs in human CRC stroma and, particularly, in the intratumoral ECs, and that this inactivation promotes tumor progression in human CRCs. 


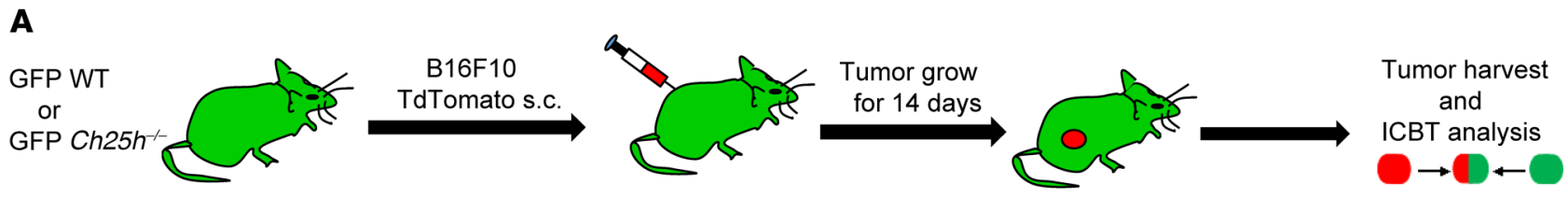

B

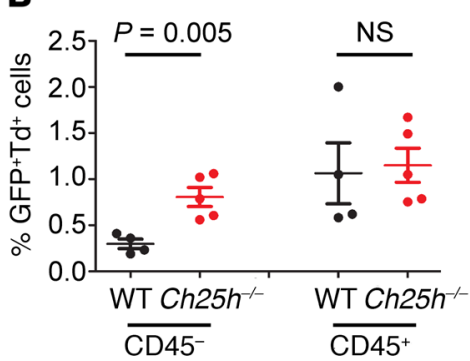

D

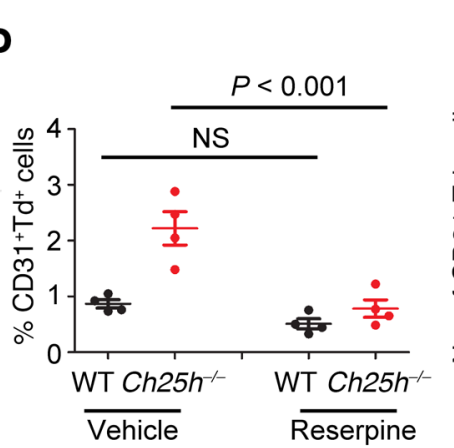

C


ICBT analysis

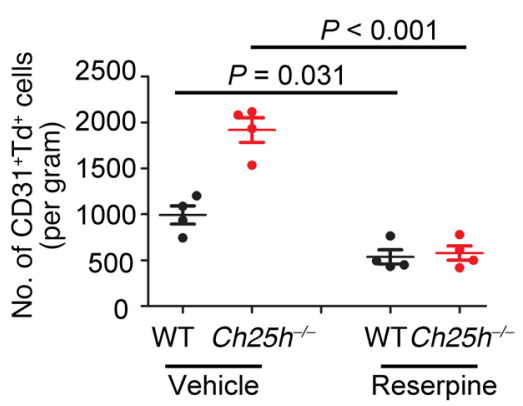

E

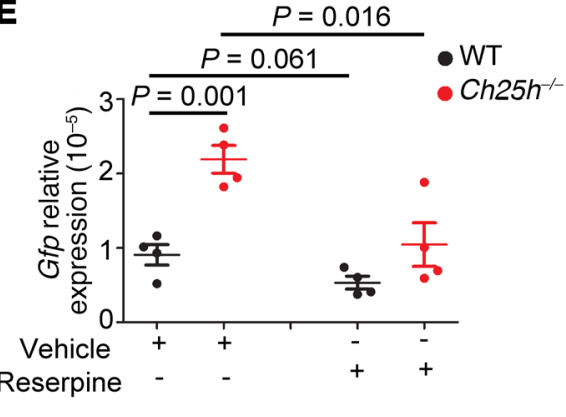

Figure 1. CH25H and reserpine control ICBT between malignant cells and endothelial cells. (A) A schematic of experiments for assessing intratumoral ICBT in vivo. (B) Flow cytometric analysis of percentage of TdTomato ${ }^{+} \mathrm{CD} 45^{-}$and TdTomato ${ }^{+} \mathrm{CD} 45^{+}$cells in the tumor microenvironment $(n=5$ for each group). (C) Flow cytometric analysis of the percentage (left) and absolute number (right) of TdTomato+CD31+ cells in tumors from GFP+ WT and GFP ${ }^{+}$ Ch25h/- mice ( $n=4-5$ for each group). (D) Flow cytometric analysis of percentage (left) and absolute number (right) of TdTomato+CD31+ cells $(n=4$ for each group) in tumors from GFP+ WT and GFP+ $C h 25 h^{-1-}$ mice administered i.p. vehicle or reserpine (1 mg/kg given every other day for 4 days). (E) qPCR analysis of Gfp mRNA in primary WT and Ch25h/- ECs after in vitro treatment with vehicle or reserpine (10 $\mu \mathrm{M}$ for 8 hours) followed by a 12-hour exposure to TEVs $(20 \mu \mathrm{g} / \mathrm{mL})$ isolated from GFP+ B16F10 cells ( $n=4$ for each group). Data are presented as mean \pm SEM. Statistical analysis was performed using 1-way ANOVA with Tukey's multiple-comparison test (B, D, and E) or 2-tailed Student's $t$ test (C). NS, not significant. Experiments were performed independently at least 3 times.

To validate these data from human patients in mouse models we examined the role of $\mathrm{CH} 25 \mathrm{H}$ in development of the intratumoral vasculature. A greater number of $\mathrm{CD} 31^{+} \mathrm{ECs}$ was found in the B16F10 melanoma tumors grown in $C h 25 h^{-/}$compared with WT mice (Figure 3, F and G). Furthermore, ablation of $\mathrm{CH} 25 \mathrm{H}$ in the tumor microenvironment resulted in a greater number and increased length of blood vessels within these tumors (Figure 3G). Similar results were obtained in subcutaneous tumors of equal size formed by pancreatic adenocarcinoma cells (Supplemental Figure 3B) or MC38 colon adenocarcinoma cells (Supplemental Figure 3C), as well as in MC38 tumors transplanted orthotopically (Supplemental Figure 3D). Analysis of functionality of these blood vessels by injection of FITC-lectin revealed that knockout of $\mathrm{CH} 25 \mathrm{H}$ increased perfusion in these tumors (Figure $3 \mathrm{H})$. In all, these results suggest that inactivation of $\mathrm{CH} 25 \mathrm{H}$ in the tumor microenvironment stimulates angiogenesis and increases tumor vascularization.

A limited screen for genes known to control tumor angiogenesis revealed a comparable level of Vegfa, Vegfr2, Tie2, Glut1, Mmp9, and Fgf1 mRNA in B16F10 tumors grown in WT and Ch25 $h^{-/}$mice (Figure 3I). Intriguingly, we detected a relatively greater expres- sion of angiopoietin-2 (Angpt2) in tumors from Ch25h/- mice compared with those from WT animals. Angpt2 was also increased in MC38 tumors from Ch25h/- mice (Figure 3I), further suggesting that inactivation of stromal $\mathrm{CH} 25 \mathrm{H}$ stimulates the expression of Angpt2 in solid tumors.

Importantly, an increase in Angpt2 induced by TEVs and reversed by reserpine was also independently detected in an RNA sequencing-based profiling of gene expression in $\mathrm{CH} 25 \mathrm{H}-$ deficient primary mouse lung ECs (Figure 4A). In addition to Angpt2, 812 out of 2998 differentially expressed genes were induced by TEVs unless pretreated with reserpine (Figure 4B). Previously implicated in transactivation of ANGPT2, transcription factors such as SP1, EGR1, GATA2, and ELF1 also exhibited altered expression after these treatments (Figure 4, A and B).

The TEV-induced increase in ANGPT2 expression and its reversal by reserpine was further validated by in vitro studies in primary mouse lung ECs. Under these conditions, treatment of Ch25h/- cells with TEVs increased Angpt2 mRNA and protein to a greater extent compared with WT ECs (Supplemental Figure 4A), whereas Angpt1 and Tie2 were not induced (Supplemental Figure $4 \mathrm{~B})$. Furthermore, TEVs induced a more robust activation of TIE2 
A

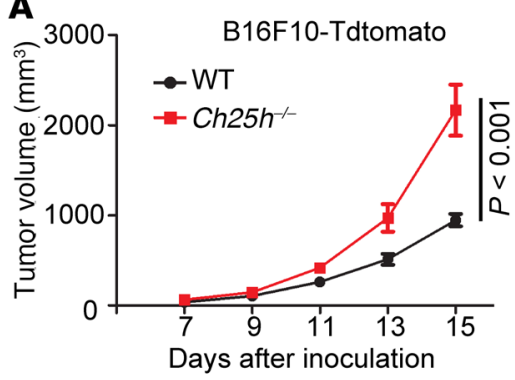

C



B







$\mathbf{E}$

WT

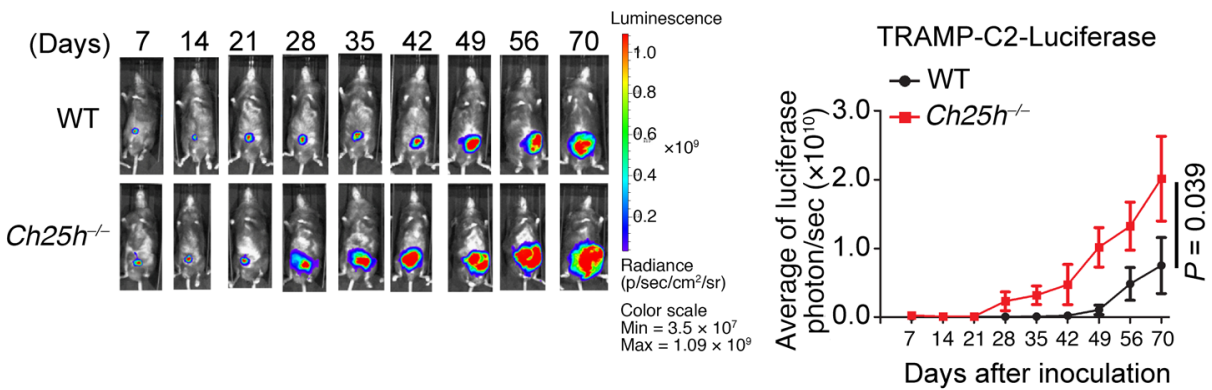

Figure 2. Stromal CH25H restricts growth of solid tumors. (A) Growth of B16F10-TdTomato tumors (inoculated s.c. at $1 \times 10^{6}$ cells $/$ mouse) in GFP+ WT and GFP' Ch25h/- mice. $n=4-5$ for each group. (B) Representative images and quantification of tumor mass on day 15 from the experiment described in panel A. (C) Growth of MH6499c4 pancreatic ductal adenocarcinoma tumors (inoculated s.c. at $1 \times 10^{5}$ cells $/$ mouse) in WT and Ch25h $h^{-1-}$ mice. $n=12-13$ for each group. (D) Growth of MC38 colon adenocarcinoma tumors (inoculated s.c. at $1 \times 10^{6}$ cells/mouse) in WT $(n=8)$ and Ch25 $h^{-/-}(n=13)$ mice. (E) Representative images and quantification of mass of MC38 colon adenocarcinoma tumors at 40 days after orthotopic inoculation of $5 \times 10^{5}$ cells into the cecum of WT $(n=5)$ or Ch25h/- $(n=4)$ mice. (F) Representative images and quantification for in vivo luciferase analysis in male WT $(n=8)$ and $C h 25 h^{-/-}(n=9)$ mice, which were inoculated into prostatic glands with TRAMP-C2-luc prostate cells $\left(1 \times 10^{6}\right)$. Data are presented as mean \pm SEM. Statistical analysis was performed using 2-way ANOVA with Tukey's multiple-comparison test (A, C, $\mathbf{D}$, and $\mathbf{F}$ ) or 2-tailed Student's $t$ test (B and $\mathbf{E})$. Experiments were performed independently at least 3 times.

in $\mathrm{Ch} 25 h^{-1-}$ ECs (Figure 4C). Importantly, pretreatment with reserpine reversed the TEV-induced increase in Angpt2 mRNA (Figure 4D) and protein (Figure 4E) in Ch25h/- ECs. These results link ICBT in ECs with induction of ANGPT2.

The angiostatic and antitumorigenic roles of endothelial $\mathrm{CH} 25 \mathrm{H}$. ANGPT2 is produced by intratumoral ECs to facilitate angiogenesis (reviewed in refs. 34-36). Given that robust induction of Angpt2 in tumors from $\mathrm{CH} 25 \mathrm{H}$-deficient mice in vivo (Figure 3I) can be faithfully recapitulated in the cultures of primary mouse lung ECs (Figure 4, D and E), we next used this in vitro system to further interrogate the importance of $\mathrm{CH} 25 \mathrm{H}$ in regulating angiogenic activity. Treatment with MC38 tumor cell-conditioned media elicited a greater increase in tube formation by the $\mathrm{Ch} 25 h^{-1-} \mathrm{ECs}$, as compared with WT cells (Figure $4 \mathrm{~F}$ and Supplemental Figure 4C). Importantly, we detected no difference in activities of WT and $C h 25 h^{-1-}$ ECs treated with vehicle, tumor cell-conditioned media lacking TEVs, or with recombinant VEGF (Figure $4 \mathrm{~F}$ and Supplemental Figure 4C). These results suggest that $\mathrm{CH} 25 \mathrm{H}$ does not indiscriminately suppress activation of ECs but specifically limits their activation by ICBT.

Indeed, treatment with purified TEVs triggered a more robust in vitro activation of $\mathrm{CH} 25 \mathrm{H}$-deficient ECs compared with their WT counterparts. These phenotypes included an increased TEV-induced proliferation manifested by either cell numbers (Figure $4 \mathrm{G}$ ) or percentage of Ki67-positive cells (Supplemental Figure 4D) and a greater migration (Supplemental Figure 4E) in Ch25 $h^{-1-}$ ECs. Furthermore, compared with WT, Ch $25 h^{-1-}$ ECs were more adept in forming endothelial tubes upon treatment with TEVs from MC38 (Figure 4H) or B16F10 (Supplemental Figure 4F) cancer cells. Importantly, neutralization of ANGPT2 prevented 
A

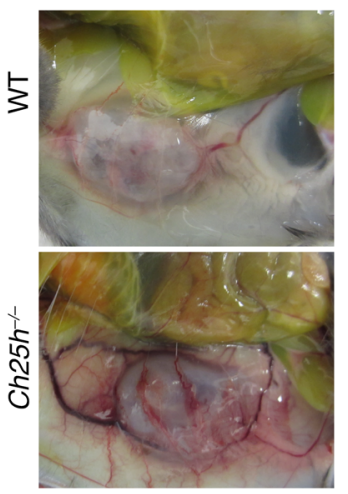

B
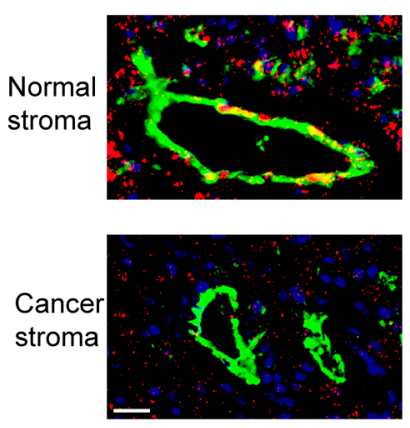

C

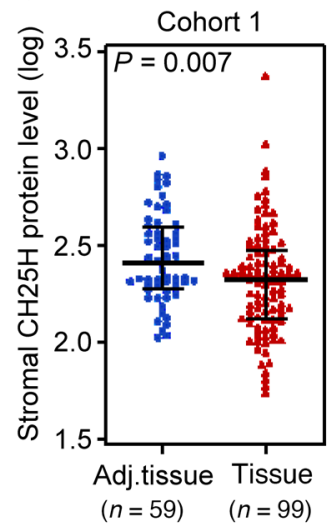

D

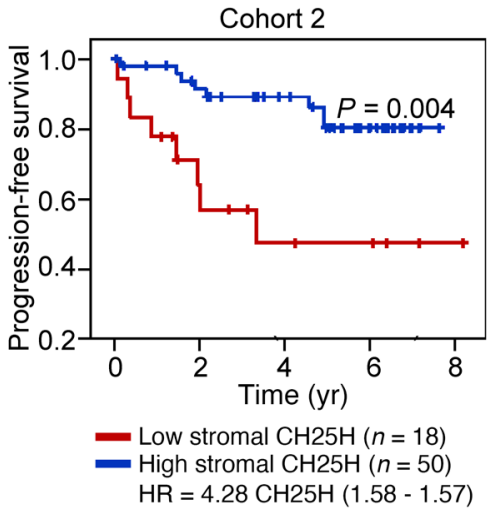

$\mathbf{E}$

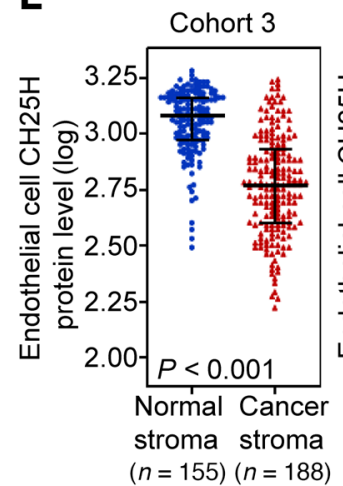

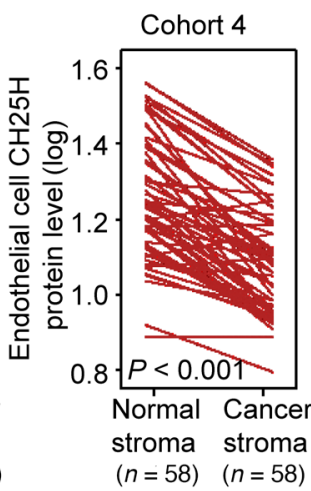

$n=58) \quad(n=58)$

$\mathbf{F}$


G
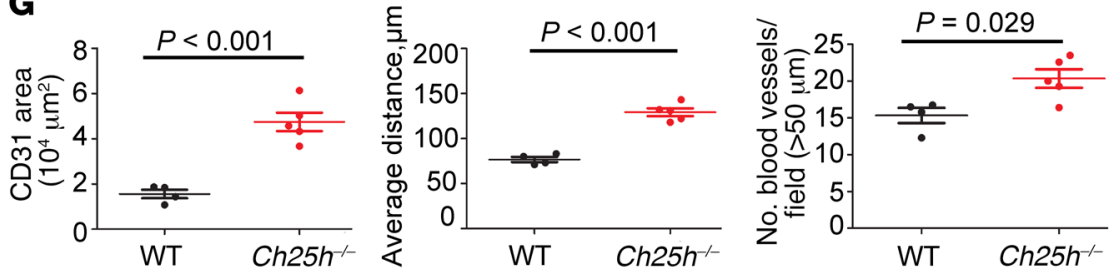
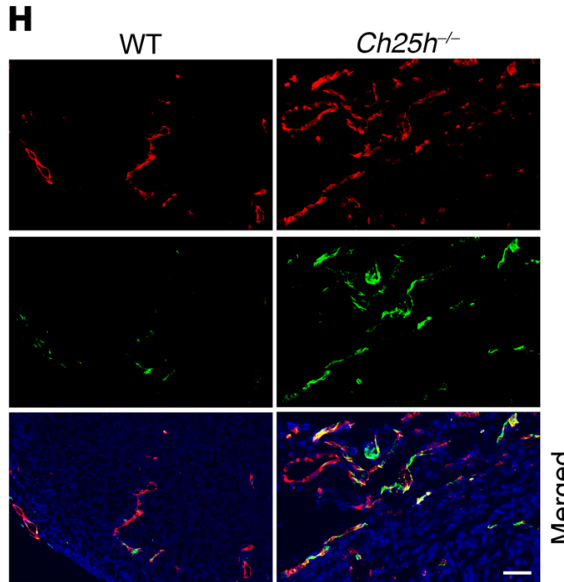

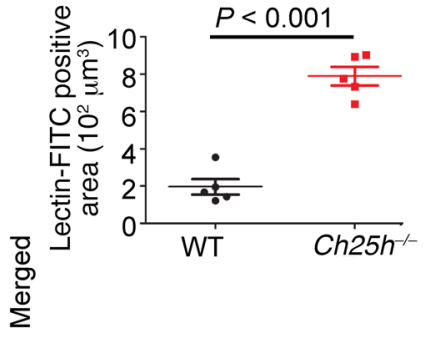

】

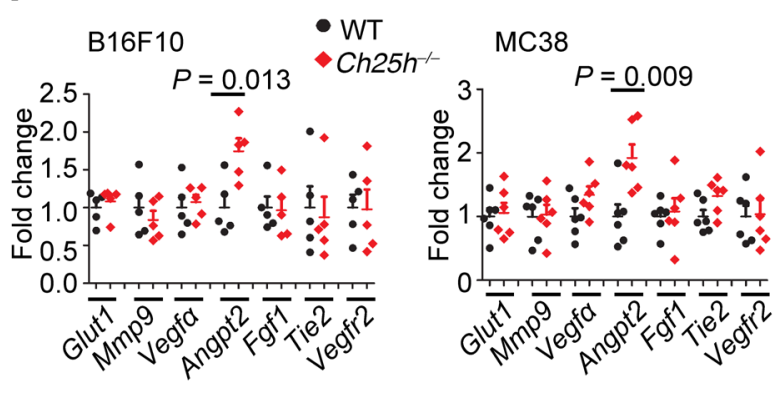

Figure 3. The angiostatic role of $\mathrm{CH} 25 \mathrm{H}$ in the tumor microenvironment. (A) A representative image of B16F10-TdTomato tumors and surrounding blood vessels in GFP+ WT and GFP+ $\mathrm{Ch}^{+} 5 \mathrm{~h}^{-1-}$ mice. (B) Representative image of blood vessels (green) and $\mathrm{CH} 25 \mathrm{H}$ (red) in normal stroma and colorectal cancer stroma. Scale bar: $100 \mu \mathrm{m}$. (C) Scatterplot of quantitative stromal CH25H protein expression levels in normal adjacent stroma and tumor stroma (Cohort 1). (D) Kaplan-Meier survival analysis of $\mathrm{CRC}$ stromal $\mathrm{CH} 25 \mathrm{H}$ protein levels, dichotomized into high and low $\mathrm{CH} 25 \mathrm{H}$ expression, indicating increased risk of recurrence with loss of $\mathrm{CH} 25 \mathrm{H}$ protein levels (Cohort 2). (E) Scatterplot of quantitative $\mathrm{CH} 25 \mathrm{H}$ protein levels within the endothelium of normal adjacent stroma and CRC tumor stroma (Cohort 3, left panel) and the validation of endothelial $\mathrm{CH} 25 \mathrm{H}$ expression levels between paired samples of normal adjacent stroma and CRC stroma (Cohort 4, right panel). (F) Analysis of CD31+ ECs in B16F10 tumors (s.c., $1 \times 10^{6}$ cells/mouse) of comparable volume grown for approximately 2 weeks in WT $(n=4)$ and $C h 25 h^{--}(n=5)$ mice. Scale bar: $100 \mu \mathrm{m}$. (C) Quantification of data from experiment described in panel F. Data averaged from 5 random fields in sections from each of 4 or 5 animals are shown. (H) Representative image (left) of colocalization of blood vessels (red) and lectin (green) area in tumor from WT and Ch25 $h^{-1-}$ mice after injection with FITC-lectin (i.v., $100 \mu \mathrm{g} /$ mouse). Quantification (right) of FITC-positive area ( $n=5$ for each group) of the images. Scale bar: $50 \mu \mathrm{m}$. (I) qPCR analysis of relative expression indicated genes in B16F10 $(n=5)$ and MC38 $(n=6)$ tumor tissues from WT and Ch25 $h^{-1-}$ mice. For each gene, mRNA levels in WT tumors were defined as 1.0. Data are presented as mean $\pm \mathrm{SEM}$. Statistical significance was determined by 2-tailed Student's $t$ test (C, E, and G-I) or log-rank (Mantel-Cox) test (D). Experiments were performed independently at least 3 times. 
A
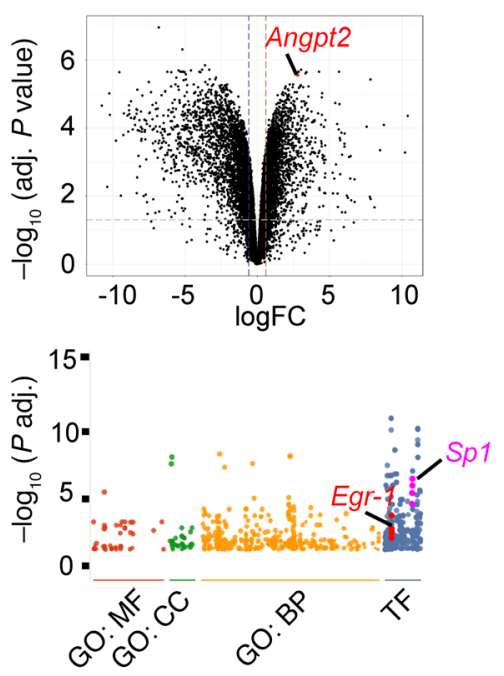

G
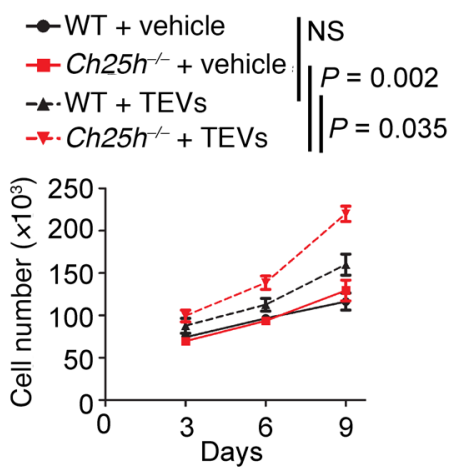

B

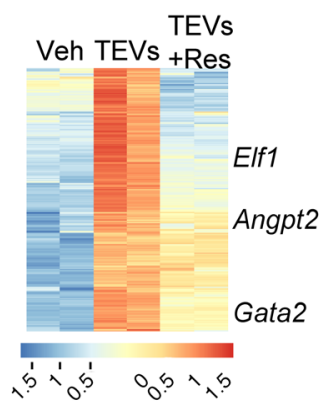

C

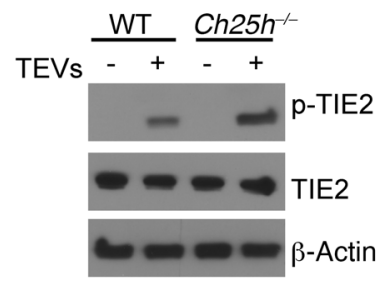

D

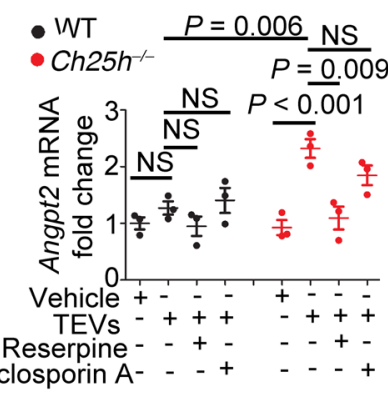



$\mathbf{F}$

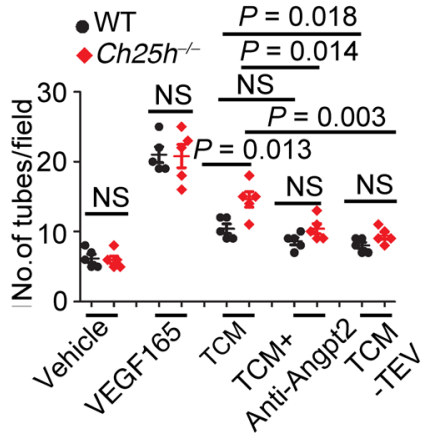

H



- WT

$\rightarrow C h 25 h^{-1}$



I

Figure 4. The ICBT-driven activation of endothelial cells is controlled by CH25H. (A) Volcano plot (upper) and Gene Ontology (CO, bottom) analyses of gene expression in Ch25h/- mouse lung ECs treated as indicated. BP, Biological Process; MF, Molecular Function; CC, Cellular Component; TF, transcription factors. (B) Heatmap analysis of gene expression from panel A. (C) Western blot analysis of TIE2 levels/phosphorylation in indicated ECs treated with MC38 TEVs (20 $\mu \mathrm{g} / \mathrm{mL}$ for 12 hours). (D) qPCR analysis of Angpt2 expression $(n=3)$ in indicated ECs pretreated with vehicle or reserpine (10 $\mu \mathrm{M}$ for 8 hours) or cyclosporin A ( $0.25 \mu \mathrm{M}$ for 24 hours) followed by PBS or TEVs $(20 \mu \mathrm{g} / \mathrm{mL}$ for 12 hours). (E) ELISA analysis of ANGPT2 in supernatant of indicated ECs from panel D. (F) Tube formation by indicated ECs treated with VEGF165 $(20 \mathrm{ng} / \mathrm{mL}$ ) or MC38 tumor cell-conditioned media (TCM) or TCM -TEV (TEV-free tumor cell-conditioned media) or TCM with addition of anti-ANGPT2 neutralizing antibody as in Supplemental Figure 4C. Data averaged from 3 random fields in each of 5 wells were quantified. (C) Proliferation of indicated ECs exposed to MC38-derived TEVs (20 $\mu \mathrm{g} / \mathrm{mL})$ for 9 days. (H) Tube formation by indicated ECs treated (or not) with MC38-derived TEVs ( $20 \mu \mathrm{g} / \mathrm{mL}$ for 12 hours) in the presence or absence of anti-ANCPT2 antibody (60 ng/mL). Representative images (left) and quantified data ( $n=5$ for each group) averaged from 3 random fields in each of the 5 wells are shown. Scale bar: $100 \mu \mathrm{m}$. (I) Tube formation by $\mathrm{Ch} 25 \mathrm{~h}^{-1-}$ ECs transduced with empty (Ctrl) or $\mathrm{CH} 25 \mathrm{H}$-expressing lentivirus (for 48 hours) or treated with vehicle or 25 -hydroxycholesterol ( $25 \mathrm{HC}, 4 \mu \mathrm{M}$ for 4 hours) and then exposed or not to MC38 TEVs ( $20 \mu \mathrm{g} / \mathrm{mL}$ for 12 hours). Data are presented as mean \pm SEM. Statistical analysis was performed by 1-way ANOVA with Tukey's multiple-comparison test (D-F, H, and I) or 2-way ANOVA with Tukey's multiple-comparison test (G). NS, not significant. Experiments were performed independently at least 3 times.

an increase in tube formation in response to tumor-conditioned media (Figure $4 \mathrm{~F}$ ) or purified TEVs (Figure $4 \mathrm{H}$ ), indicating that ANGPT2 is required for the ICBT-induced activation of ECs. Furthermore, ICBT-driven hyperactivation of Ch25h/- ECs could be effectively reversed by either reexpressing $\mathrm{CH} 25 \mathrm{H}$ or by treating these cells with the end-product of the $\mathrm{CH} 25 \mathrm{H}$ enzymatic activ- ity $-25 \mathrm{HC}$ (Figure 4I). Collectively, these results suggest that $\mathrm{CH} 25 \mathrm{H}$ acts as a biological barrier that restricts the ICBT-induced ANGPT2-dependent activation of ECs.

These results, together with the protumorigenic phenotypes observed in $\mathrm{Ch} 25 \mathrm{~h}^{-/-}$mice (Figure 2), prompted us to concentrate on specific roles of endothelial $\mathrm{CH} 25 \mathrm{H}$ in vivo. To this end, we iso- 
A

GFP
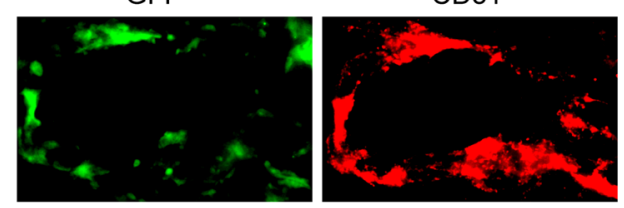

C
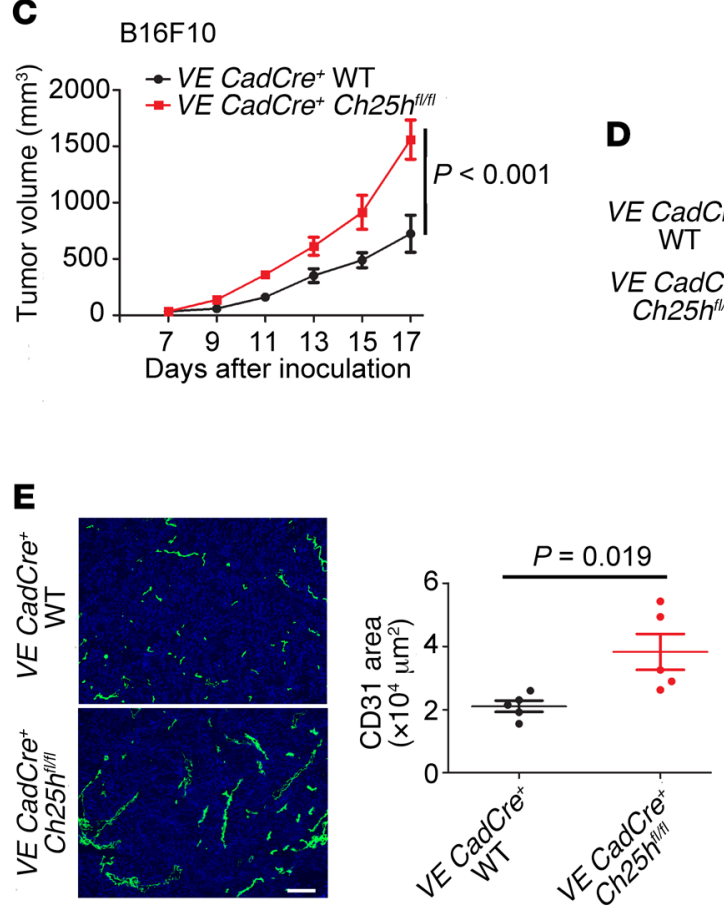

B
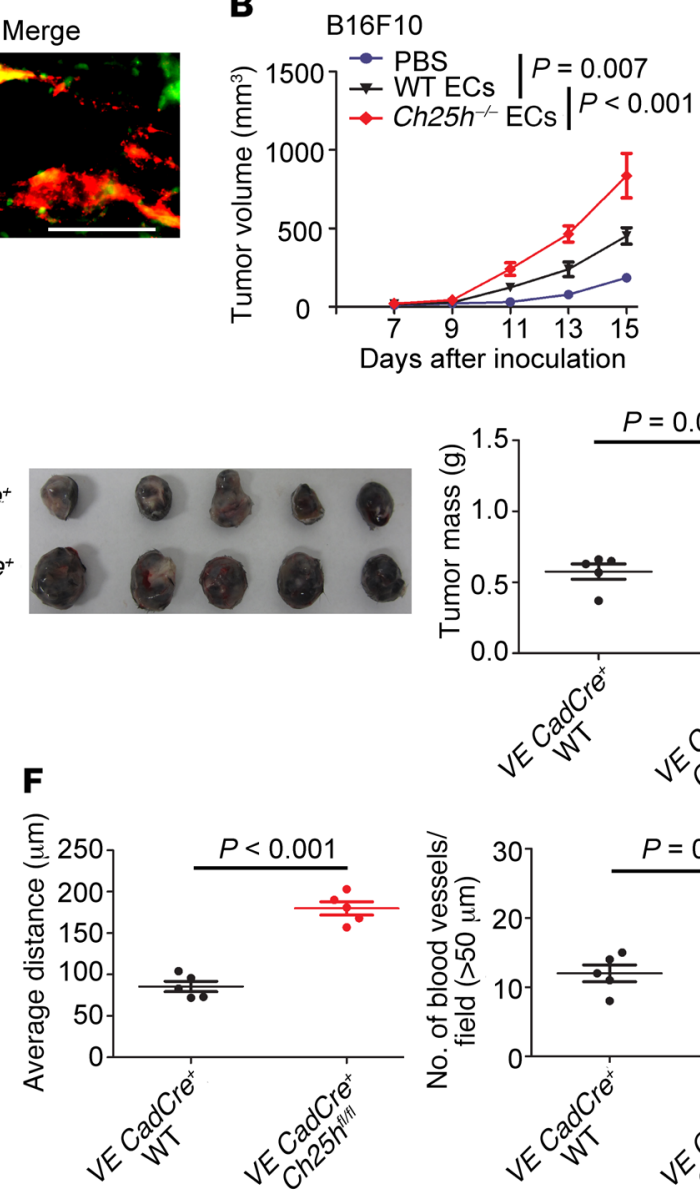

Figure 5. Endothelial expression of $\mathrm{CH} 25 \mathrm{H}$ drives its angiostatic and antitumorigenic functions in vivo. (A) Representative images of colocalization of GFP-expressing Ch25h/- ECs (green) with blood vessels (red). Scale bar: $20 \mu \mathrm{m}$. (B) Analysis of growth of tumors formed in WT host mice by B16F10 malignant cells $\left(3 \times 10^{5} /\right.$ mouse) coinjected s.c. with vehicle $(n=4)$ or with primary lung ECs $\left(6 \times 10^{4} /\right.$ mouse) isolated from WT or Ch $25 h^{-/-}$mice $(n=5$ for both groups). (C) Analysis of growth of B16F10 tumors (s.c., $1 \times 10^{6}$ cells/mouse) in VE-cadherin-Cre ${ }^{+}$WT and VE-cadherin-Cre ${ }^{+}$Ch2 $25 h^{f / f f l}$ mice $(n=5$ for each group). (D) Representative images and B16F10 tumor mass analysis from experiment described in panel C (tumors harvested on day 17 after inoculation). (E) Representative immunofluorescence images of CD31 staining of B16F10 tumors from VE-cadherin-Cre+ WT and VE-cadherin-Cre+ Ch25 ${ }^{f l / f l}$ mice (left) and quantification of CD31-positive areas (right). Quantification averaged from 5 random fields in sections from each of 5 animals is shown. Scale bar: 100 $\mu \mathrm{m}$. (F) Quantification of average length and number of blood vessels $(>50 \mu \mathrm{m})$ from experiment shown in panel E. Data averaged from 5 random fields in sections from each of 5 animals are shown. Data are shown as mean \pm SEM. Statistical analysis was performed using 2-way ANOVA with Tukey's multiplecomparison test (B and $\mathbf{C}$ ) or 2-tailed Student's $t$ test (D-F). Experiments were performed independently at least 3 times.

lated ECs from the lungs of naive $\mathrm{GFP}^{+} \mathrm{WT}$ or $\mathrm{Ch} 25 h^{-1}$ mice and coinjected these cells with B16F10 cells into the flanks of WT mice. Under these conditions, at least some of the transferred $\mathrm{GFP}^{+}$ ECs were incorporated into intratumoral blood vessels (Figure $5 \mathrm{~A})$. Whereas ECs of both genotypes accelerated tumor growth, Ch $25 h^{-1-}$ ECs were significantly more active in these settings (Figure 5B and Supplemental Figure 5A), indicating that endothelial $\mathrm{CH} 25 \mathrm{H}$ interferes with the ability of ECs to support tumor growth.

To further test this hypothesis, we generated a conditional knockout allele of Ch25h by flanking the sole exon of this gene with loxP sites (Supplemental Figure 5B). We crossed these mice with animals that express Cre recombinase under the EC-specific VE-cadherin promoter (37); the resulting animals lacked $\mathrm{CH} 25 \mathrm{H}$ in primary lung ECs but not fibroblasts (Supplemental Figure 5C). Whereas no obvious vascular alterations or other abnormalities were detected in these naive animals, they displayed a notable phenotype when challenged with subcutaneous tumors. Under these conditions, efficient ablation of $\mathrm{CH} 25 \mathrm{H}$ was achieved in the intratumoral ECs (Supplemental Figure 5D). Importantly, growth of B16F10 melanoma tumors was significantly accelerated in mice lacking $\mathrm{CH} 25 \mathrm{H}$ in the ECs (Figure 5, C and D). Furthermore, ablation of $\mathrm{CH} 25 \mathrm{H}$ in ECs notably increased intratumoral angiogenesis, as evident from an increased $\mathrm{CD} 31^{+}$area as well as a greater number of blood vessels and an increase in their length (Figure 5, $\mathrm{E}$ and F). Similar observations were made in experiments involving MC38 colon adenocarcinoma tumors (Supplemental Figure 5, $\mathrm{E}-\mathrm{H})$. In all, these results suggest that endothelial $\mathrm{CH} 25 \mathrm{H}$ plays important angiostatic and antitumorigenic roles.

Reserpine inhibits tumor angiogenesis and improves the outcome of radio- and chemotherapies. We have previously reported that treatment with reserpine increases the expression of $\mathrm{CH} 25 \mathrm{H}$ in TEV-treated cells (26). Thus, we utilized this agent as a complementary pharmacologic tool to ascertain the importance of ICBT-driven endothelial activation and tumor angiogenesis. 
Used at previously described low doses (26), reserpine was well tolerated and did not cause sleepiness or decrease animal weight while inhibiting the intratumoral ICBT (Figure 1, D and E). Furthermore, in vitro pretreatment of ECs with reserpine suppressed TEV-induced expression of Angpt2 (Figure 4, B, D, and E) and significantly inhibited endothelial tube formation (Supplemental Figure 6A). These results prompted us to examine the effect of reserpine on tumor angiogenesis in vivo.

Administration of reserpine did not decrease expression of Angpt1 or Tie2 in the B16F10 tumors growing in WT or Ch25h ${ }^{-1}$ mice (Supplemental Figure 6B). However, this treatment abolished an increase in expression of Angpt2 (Figure 6A) and notably suppressed angiogenesis (Figure 6B) in tumors from Ch $25 h^{-/-}$mice. Similar results were also obtained in animals bearing MC38 colon tumors (Supplemental Figure 6C), indicating that reserpine exhibits a robust angiostatic effect in solid tumors.

Suppressive effects of reserpine on angiogenesis and growth were seen in human HCT116 colon tumors growing in the immune-deficient mice (Figure 6, C and D). Likewise in the immune-competent settings, administration of reserpine inhibited B16F10 tumor growth in both WT and Ch25 $h^{-/}$mice (Figure $6, \mathrm{E}$ and $\mathrm{F}$ ). This effect was also observed in mice harboring the VE-cadherin-Cre allele in the $C h 25 h^{+/+}$or $C h 25 h^{f / f l}$ background (Figure 6G) as well as in Ch25 $h^{-/-}$mice inoculated with MC38 tumors (Supplemental Figure 6, D and E).

Expression of ANGPT2 is thought to undermine the efficacy of antiangiogenic therapies targeting VEGF (bevacizumab) or its receptor (sunitinib) $(35,38-41)$. Given that reserpine suppresses the ICBT-induced production of ANGPT2 (Figures 4 and 6A), we hoped to optimize the antiangiogenic therapy by combining these agents (Figure 7A). Indeed, adding reserpine to the sunitinib regimen decreased plasma levels of ANGPT2 (Supplemental Figure $7 \mathrm{~A}$ ), maximized the suppression of angiogenesis (Figure 7B), and augmented the antitumorigenic effects in mice bearing MC38 (Figure 7, C and D) or B16F10 tumors (Supplemental Figure 7B). These effects of reserpine were similar to those of rebastinib, a bona fide inhibitor of the ANGPT2 pathway (Supplemental Figure 7, C-G). Collectively, these results indicate that reserpine alone or in combination with agents targeting VEGF can be used as a pharmacologic agent to suppress the intratumoral angiogenesis and inhibit growth of primary solid tumors.

Reserpine was previously included in the screen for agents affecting TEV uptake (26) based on its activity as a vesicular reuptake inhibitor (reviewed in ref. 42). Intriguingly, in addition to inhibiting TEV uptake by splenocytes or ECs (ref. 26 and Figure $1 \mathrm{E}$ and Supplemental Figure 1, J and K), we noticed a significant suppressive effect of reserpine on both protein content and numbers of TEVs produced by B16F10 or MC38 cancer cells in vitro (Figure 8A). Furthermore, treatment of MC38 cells with reserpine decreased the expression of several genes including Rab11b, Rab27a, Ykt6, and Snap23 (Figure 8B), which play an important role in formation and/or uptake of extracellular vesicles $(43,44)$.

Preincubation of DiD-labeled TEVs with reserpine did not affect transfer of DiD into ECs (Supplemental Figure 8A), suggesting that ECs themselves are targets for reserpine-elicited uptake inhibition. Importantly, given that reserpine upregulates $\mathrm{CH} 25 \mathrm{H}$ expression (26) and following an analogy with $25 \mathrm{HC}$, which alters membrane fluidity and inhibits lipid membrane fusion (32), we analyzed the effects of reserpine on membrane fluidity using 1,6-diphenyl-1,3,5-hexatriene as a sensor of the bilayer membrane structure alterations. Polarization of this biosensor was increased by reserpine (Figure $8 \mathrm{C}$ ), indicating that reserpine can increase the rigidity of EC membranes. This possibility was further tested by studies involving transfer of DiD dye to ECs from liposomes that lack any protein molecules on their surface; reserpine suppressed this transfer (Figure $8 \mathrm{D}$ and Supplemental Figure $8 \mathrm{~B}$ ). These results collectively suggest that mechanisms of ICBT inhibition by reserpine include its effect on the EC lipid membranes.

A yet greater impetus for the use of reserpine to suppress ICBT came from the observations that chemo- and radiotherapies activate the formation and release of prometastatic TEVs by primary tumors $(24,25)$; this activation contributes to resistance $(5,7,19$, $45)$ as well as to therapy-triggered stimulation of metastatic disease (22-24). We next sought to determine whether reserpine addition can improve efficacies of chemo-/radiotherapies while preventing their negative prometastatic effects.

Consistent with suppression of TEV production in vitro (Figure $8 \mathrm{~A}$ ), in vivo administration of reserpine decreased the amount of the exosomal marker CD63 in the plasma from the B16F10 or MC38 tumor-bearing mice subjected or not to ionizing radiation or chemotherapy treatment (Figure 9A and Supplemental Figure 9A). Likewise, reserpine treatment abolished an increase in the numbers of TEVs found in plasma of mice subjected to chemotherapy (Supplemental Figure 9A). Given that reserpine can also inhibit TEV uptake (26), these data collectively suggest that reserpine can suppress ICBT through multiple mechanisms.

Combining reserpine treatment with radiation therapy augmented the inhibition of subcutaneous primary B16F10 tumor growth and prolonged animal survival (Figure 9, B and C). Importantly, reserpine also dramatically suppressed the appearance of distant metastatic melanoma lesions in the lungs and prevented an increase in the number of these lesions in response to irradiation of primary tumors (Figure 9D and Supplemental Figure 9B).

The effects of reserpine were next determined in the chemotherapy setting, in which orthotopically inoculated MC38 colon adenocarcinomas were treated with the FOLFOX regimen (Figure 9E). This regimen, which includes oxaliplatin, folinic acid, and 5-fluorouracil, is standard for treatment of patients with metastatic colorectal cancers and is often combined with antiangiogenic agents (46). Intriguingly, 5-fluorouracil was also implicated in stimulation of postliminary metastatic disease (47) and in the induction of genes associated with poor prognosis in a cohort of patients that received FOLFOX (48). In our preclinical model, the inclusion of reserpine notably decreased the intratumoral angiogenesis otherwise stimulated by the FOLFOX regimen (Supplemental Figure 9, C and D). Importantly, while being well tolerated (ref. 26 and Supplemental Figure 9E), reserpine also potentiated therapeutic effects of FOLFOX on primary tumors (Figure 9F), robustly suppressed metastases into liver (Figure 9G), and decreased the number of local lesions in the gut (Supplemental Figure 9F). Collectively, these data provide the rationale for the inclusion of reserpine into the radio-/chemotherapy regimens to increase their overall efficacy and, most importantly, to prevent the prometastatic side effects of such treatments. 
A

- WT

- Ch25h-r

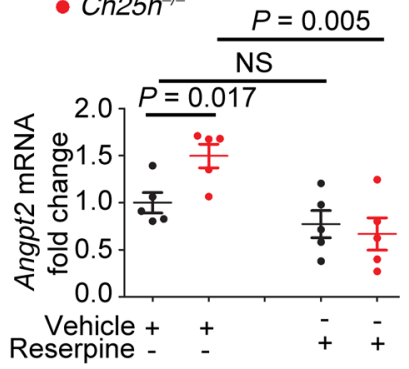

C

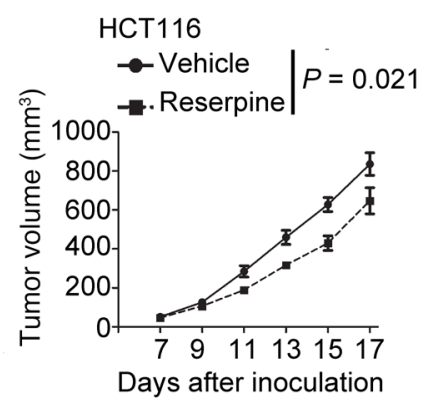

$\mathbf{F}$

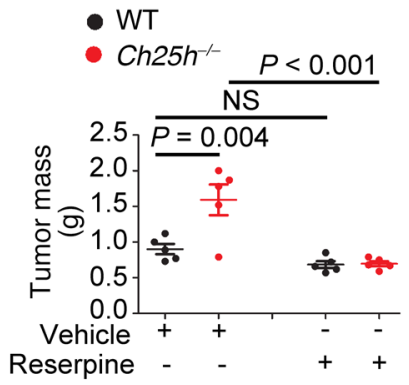

B


D
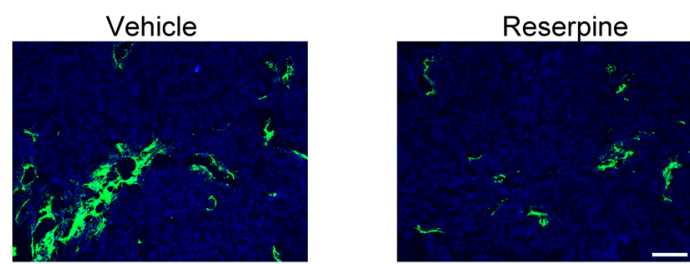

$\mathbf{E}$
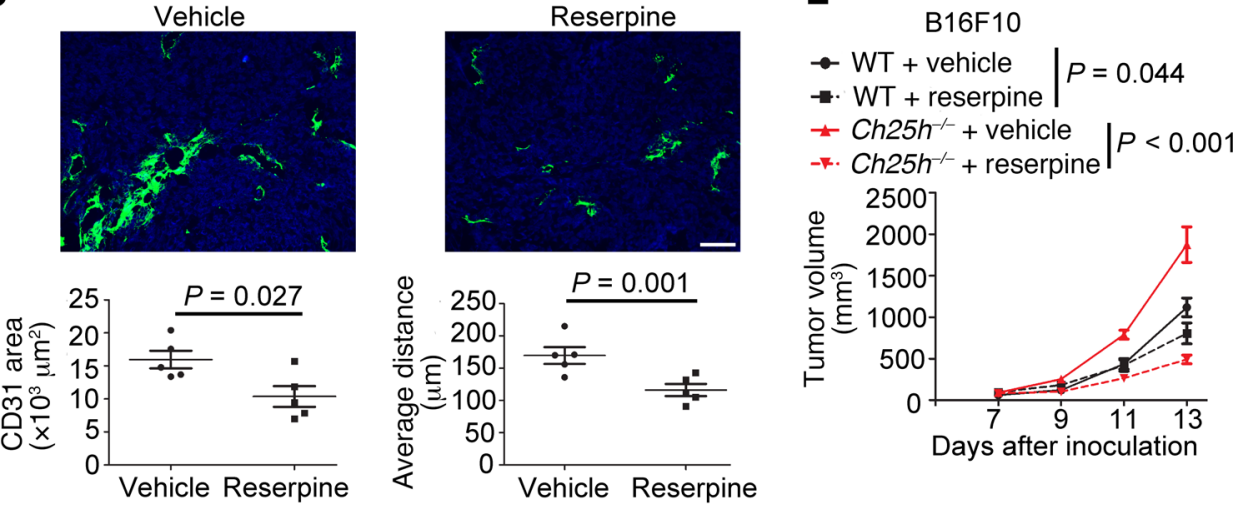

G

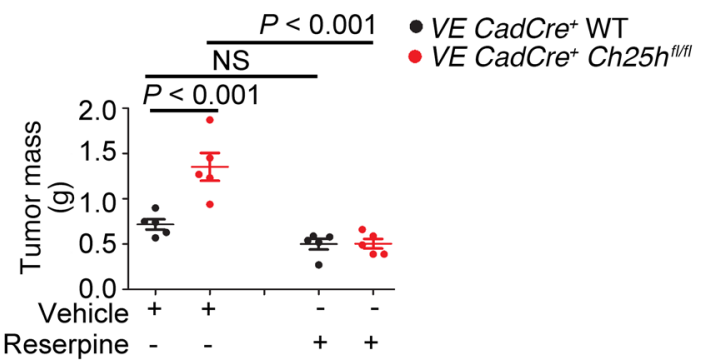

Figure 6. Angiostatic and antitumorigenic effects of reserpine in solid tumors. (A) qPCR analysis of relative Angpt2 mRNA levels in B16F10 tumors from WT and Ch $25 h^{-/-}$mice treated with vehicle or reserpine ( $1 \mathrm{mg} / \mathrm{kg}$, i.p. every other day for 4 days). $n=5$ for each group. (B) Representative immunofluorescence images and quantification of CD31-positive areas in B16F10 tumors from WT and Ch25 $h^{-/-}$mice $(n=5$ for each group) treated with vehicle or reserpine as described in panel A. Scale bar: $100 \mu \mathrm{m}$. (C) Growth of human HCT116 tumors (inoculated s.c. at $5 \times 10^{6}$ cells/mouse) in NSG mice treated with vehicle or reserpine $(1 \mathrm{mg} / \mathrm{kg}$ ) every other day. $n=5$ for each group. (D) Representative immunofluorescence image (upper) of CD31 staining of HCT116 tumors from NSG mice treated with vehicle or reserpine. Quantification (bottom) of CD31-positive areas and average distance of blood vessels. Quantification averaged from 5 random fields in sections from each of 5 animals is shown ( $n=5$ for each group). Scale bar: $100 \mu \mathrm{m}$. (E) Analysis of B16F10 tumor growth (inoculated s.c. at $1 \times 10^{6}$ cells/mouse) in WT and Ch25h/- mice ( $n=5$ for each group) followed by vehicle or reserpine treatment $(1 \mathrm{mg} / \mathrm{kg}$, i.p. every other day). (F) Analysis of B16F10 tumor mass on day 15 of the experiment described in panel E. (G) Analysis of B16F10 tumor mass on day 15 after inoculation (s.c. at $1 \times 10^{6}$ cells/mouse) into indicated mice ( $n=5$ for each group) followed by vehicle or reserpine treatment $(1 \mathrm{mg} / \mathrm{kg}$, i.p. every other day). Data are presented as mean \pm SEM. Statistical analysis was performed using 1-way ANOVA with Tukey's multiple-comparison test (A, B, F, and G), 2-way ANOVA with Tukey's multiple-comparison test (C and E), or 2-tailed Student's $t$ test (D). NS, not significant. Experiments were performed independently at least 3 times.

\section{Discussion}

Endothelial CH25H as a genetic suppressor of ICBT-driven intratumoral angiogenesis. Given the paramount role of ICBT in tumor growth, progression, and resistance to therapies (5-7), it is of critical importance to understand how ICBT is regulated and could be pharmacologically controlled in vivo. An increased ICBT between malignant cells and $\mathrm{CH} 25 \mathrm{H}$-deficient ECs (Figure 1) might be reflective of ECs representing the first barrier encountered by the circulating tumor-derived matter. $\mathrm{CH} 25 \mathrm{H}$ is induced by IFN, and downregulation of its receptor often occurs in many types of cancer $(28,49-51)$ and contributes to angiogenic activation by VEGF (52). Importantly, the loss of $\mathrm{CH} 25 \mathrm{H}$ per se does not 
A

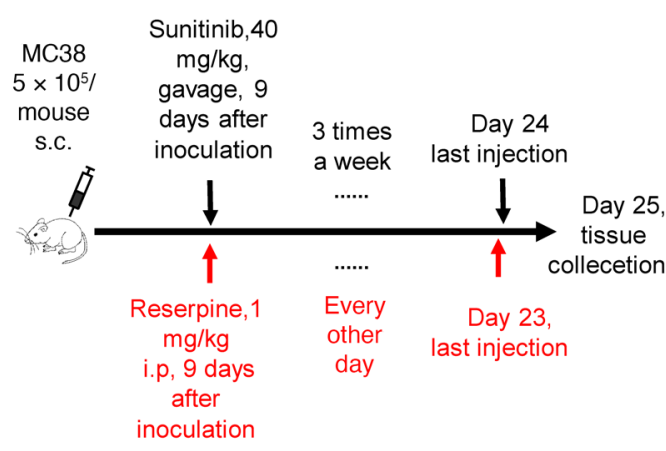

C

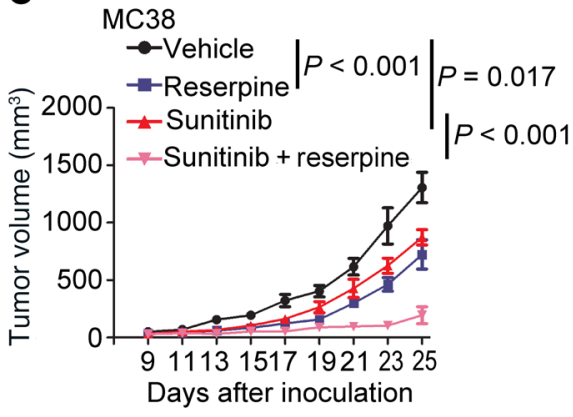

D
B

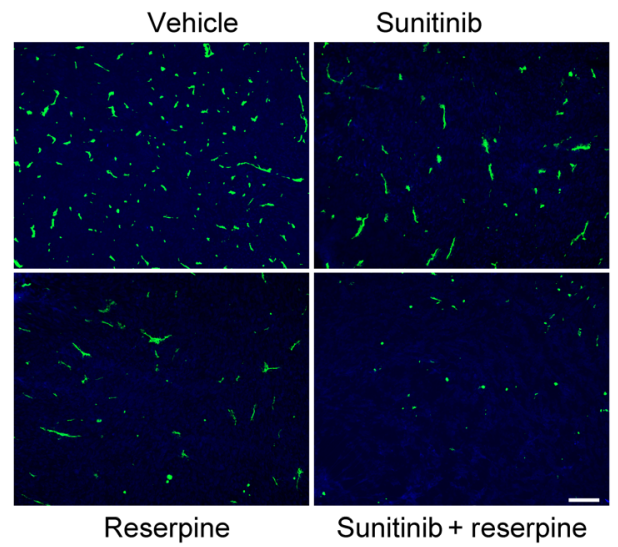

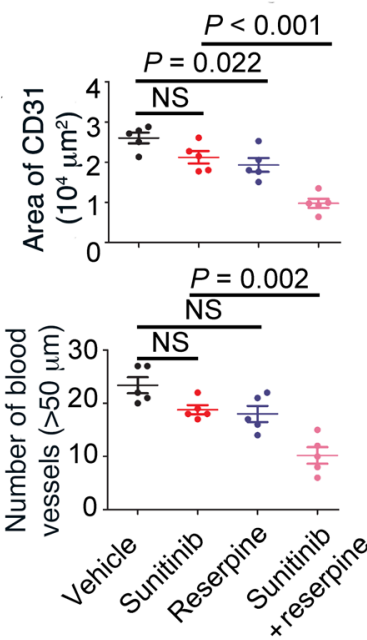
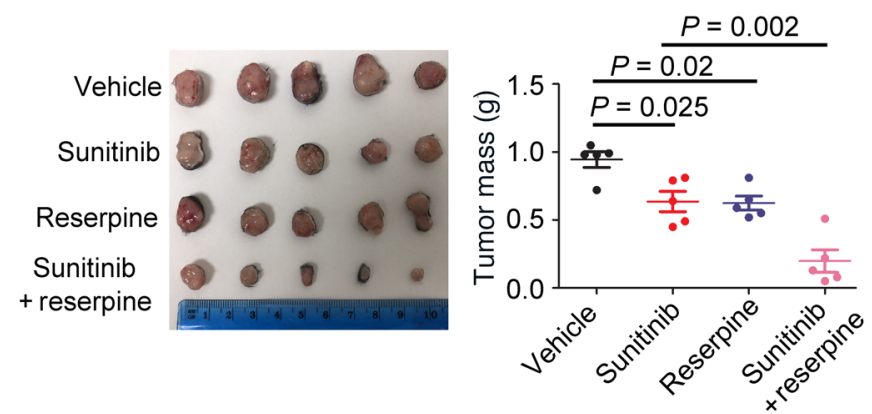

Figure 7. Combination of reserpine and antiangiogenic therapy. (A) Schematic of treatment of MC38 tumor-bearing mice with sunitinib, reserpine, or their combination. (B) Representative immunofluorescence images and quantification of angiogenesis parameters in MC38 tumors from WT mice treated as in panel A ( $n=5$ for each group). Scale bar: $100 \mu \mathrm{m}$. (C) Analysis of MC38 tumor volume in WT mice treated as in panel A ( $n=5$ for each group). (D) Analysis of mass of MC38 tumors in WT mice on day 25 of the experiment described in panel C ( $n=5$ for each group). Data are presented as mean \pm SEM. Statistical analysis was performed using 1-way ANOVA with Tukey's multiple-comparison test (B and D) or 2-way ANOVA with Tukey's multiple-comparison test (C). NS, not significant. Experiments were performed independently at least 3 times.

stimulate ECs; however, CH25H-deficient ECs are more sensitive to TEV-induced activation of ECs. Although the importance of $\mathrm{CH} 25 \mathrm{H}$ in regulating ICBT in other cell types should not be ruled out, our data specifically characterize endothelial $\mathrm{CH} 25 \mathrm{H}$ as a key suppressor of the ICBT-induced upregulation of ANGPT2, activation of ECs, and intratumoral angiogenesis.

$\mathrm{CH} 25 \mathrm{H}$ catalyzes monooxygenation of cholesterol into $25 \mathrm{HC}$. Its absence may promote formation of other types of oxycholesterols such as proangiogenic 27-hydroxycholesterol (53). Nevertheless, evidence demonstrating the ability of $25 \mathrm{HC}$ to directly suppress tube formation (Figure 4I) and EC proliferation (54) suggests a key role for $25 \mathrm{HC}$ in the angiostatic phenotype. It is important to note that $25 \mathrm{HC}$ inhibits lipid membrane fusion (32), which is essential for cell fusion, uptake of TEVs and apoptotic bodies, cell junction and tunneling nanotube formation, and other events enabling ICBT (reviewed in ref. 9). Whereas the importance of $\mathrm{CH} 25 \mathrm{H}$ and $25 \mathrm{HC}$ in restricting the uptake of TEVs has been demonstrated (26), future studies will focus on determining specific roles of these regulators in other mechanisms of ICBT.

Numerous mediators of angiogenesis delivered to ECs by extracellular vesicles from either normal or malignant cells include VEGF-A and -D, WNT4, IL-8, carbonic anhydrase 9, diverse types of noncoding RNAs, and others (55-62). It is likely that more than one type of these diverse biomolecules is redundantly responsible for increased ANGPT2 and resulting angiogenesis in the absence of endothelial $\mathrm{CH} 25 \mathrm{H}$. Under these conditions, induction of ANGPT2 upon inactivation of $\mathrm{CH} 25 \mathrm{H}$ is likely triggered by an increased extent of ICBT as well as by additional $\mathrm{CH} 25 \mathrm{H}$-dependent mechanisms that affect TEVsensitive and/or reserpine-sensitive transcription factors such as SP1, EGR1, ELF1, and GATA2 and related pathways uncovered in our study (Figure 4, A and B). Intriguingly, cyclosporin A, an inhibitor of the calcineurin/NFAT/ANGPT2 signaling axis (63), also had a modest effect on ANGPT2 induction by TEVs (Figure 4D), suggesting that diverse and likely redundant pathways mediate the effects of ICBT.

It is likely that the importance of endothelial $\mathrm{CH} 25 \mathrm{H}$ for suppressing tumor angiogenesis and growth gleaned from experiments using knockout models is seriously underestimated because of notable decreases in $\mathrm{CH} 25 \mathrm{H}$ levels in the intratumoral ECs, as detected in human CRC patients (Figure 3, B-D). This downregulation of $\mathrm{CH} 25 \mathrm{H}$ in the tumor microenvironment was significantly associated with poor prognosis (Figure 3D) and could be mediated by downregulation of $\mathrm{CH} 25 \mathrm{H}$ expression in response to TEVs (26) as well as by additional, yet to be delineated mechanisms.

Reserpine as a pharmacologic inhibitor of ICBT and, potentially, a component of anticancer therapies. Importantly, the ICBT-driven ANGPT2 expression, EC activation, and intratumoral angiogene- 
A
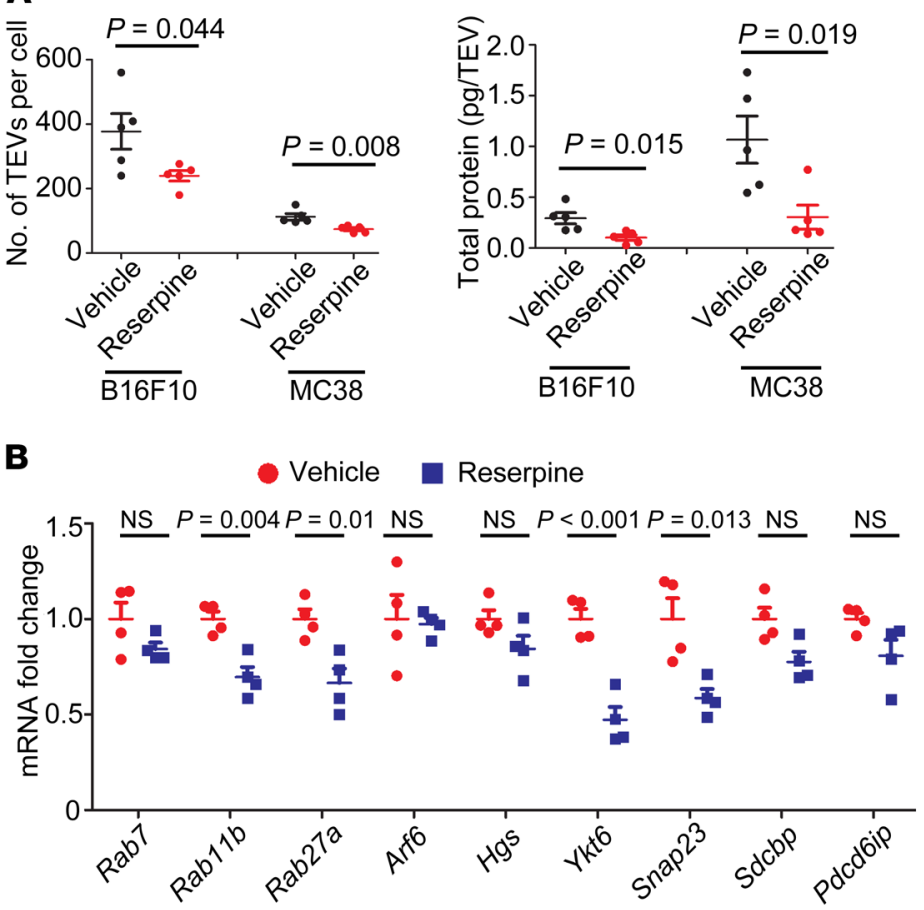

C
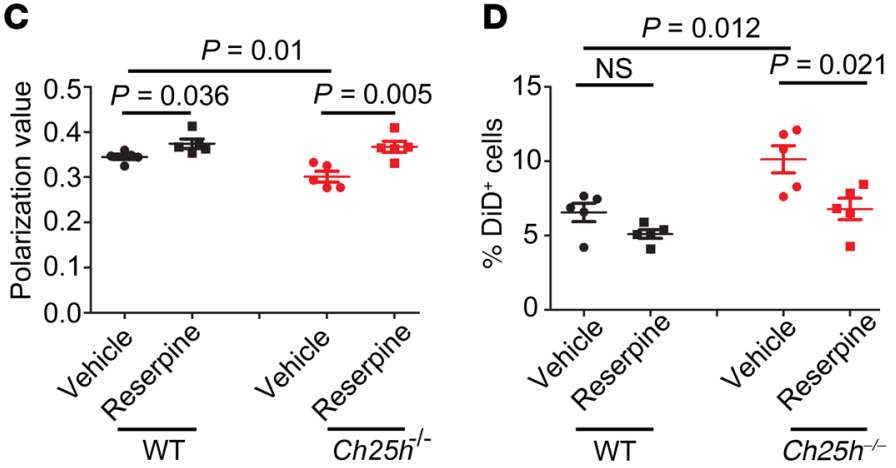

Figure 8. Mechanism of reserpine-mediated TEV uptake inhibition. (A) Quantification of numbers (upper panel) and total protein content (bottom panel) of TEVs released by the indicated cells following treatment with vehicle or reserpine (10 $\mu \mathrm{M}$ for 48 hours) in vitro. (B) qPCR analysis of Rab7, Rab11b, Rab27a, Sdcbp, Arf6, Ykt6, Snap23, Hgs, and Pdcd6ip relative levels ( $n=4$ for each group) in MC38 cancer cells treated with vehicle or reserpine (10 $\mu \mathrm{M}$ for 12 hours). (C) Analysis of plasma membrane polarization in WT and Ch25h/- ECs treated with reserpine $(10 \mu \mathrm{M})$ for 12 hours. (D) Flow cytometric analysis of percentage of DiD+CD31+ cells upon incubation of indicated ECs with DiD-labeled liposomes (1 $\mu \mathrm{g} / \mathrm{mL})$ in the presence or absence of reserpine $(10 \mu \mathrm{M})$ for 8 hours $(n=5$ for each group). Data are presented as mean \pm SEM. Statistical analysis was performed using 1-way ANOVA with Tukey's multiple-comparison test (A, C, and $\mathbf{D})$ or 2-tailed Student's $t$ test (B). NS, not significant. Experiments were performed independently at least 3 times.

sis can be virtually nullified by treatment with reserpine (Figures 4 and 6). Given that production of ANGPT2 is implicated in clinical refractoriness to the antiangiogenic therapies involving bevacizumab $(38,39)$, reserpine can be employed to specifically suppress the ICBT-stimulated ANGPT2-dependent intratumoral angiogenesis and complement agents targeting the VEGF pathway, similarly to TIE2 pathway inhibitors such as rebastinib (Figure 6 and Supplemental Figure 6).
Perhaps even more exciting is the potential for clinical use of reserpine to improve the efficacy of other types of anticancer treatment, including chemo- and radiotherapies supported by the preclinical data presented here (Figure 9). Reserpine has been widely used as a drug for treatment of hypertension and has also been chosen for its ability to inhibit vesicular monoamine transporter-2 and vesicular reuptake (42). We have previously reported that treatment with reserpine increases the expression of $\mathrm{CH} 25 \mathrm{H}$ in TEV-treated cells (26). Here we show that reserpine limits the ICBT between malignant and benign cells in vivo (Figure 1) by likely more than one mechanism, including suppression of TEV uptake (26) and a decrease in circulating TEVs (Figure 9A and Supplemental Figure 9A), which can be plausibly attributed to altered TEV production/loading in vitro (Figure 8A). Molecular mechanisms underlying the inhibition of ICBT by reserpine are likely to involve effects on expression of genes involved in production of extracellular vesicles and on plasma membrane fluidity and its ability to fuse with lipid membrane vesicles (Figure 8, B-D). Potential effects of reserpine on additional modes of ICBT (e.g., cell fusion or formation of tunneling nanotubes) require further studies.

Although reserpine elicited only a modest effect on proliferation of malignant cells in vitro (26), additional ICBTindependent mechanisms by which this agent may help to suppress angiogenesis and growth of solid tumors cannot be excluded. Regardless of these specific mechanisms, it is important to note that reserpine robustly suppressed the increase in metastatic disease triggered by therapeutic regimens mostly designed to efficiently eradicate primary tumors (Figure 9).

The relatively low cost of reserpine therapy adds to its benefits, which could be especially important to battle metastatic disease in economically disadvantaged patients worldwide. Given that reserpine is well tolerated and has already been approved for use in human patients, this drug can be immediately tested in clinical trials for inclusion in standard regimens used in the treatment of solid tumors. Furthermore, future development of novel means to control ICBT and improve the outcome of anticancer therapies hold additional promise.

\section{Methods}

Detailed methods can be found in the supplemental material.

Human CRC specimens and their analyses. Human CRC tissue microarrays, consisting of formalin-fixed, paraffin-embedded tissue cores, were stained for $\mathrm{CH} 25 \mathrm{H}$. The detailed description of Cohorts 1-4 is provided in the supplemental material. $\mathrm{CH} 25 \mathrm{H}, \mathrm{CD} 31$, and cytokeratin detection was performed using immunofluorescence and immunohistochemistry, as previously described (64). Quantitative biomarker analysis was performed using Tissue Studio image analysis software (Definiens) to identify epithelial or stromal regions, facilitated by DAPI-stained cell nuclei and cytokeratinstained cancer cells.

Animal studies. Besides NSG, all other mouse strains (including WT, Ch25h/-, TgN(ActbEGFP)1Osb/J, and VE-cadherin-Cre (65) purchased from The Jackson Laboratory were on the C57BL/6J background. The conditional $C h 25 h$ allele was created by flanking the single exon of the Ch25h gene with loxP sites inserted into the noncon- 
A



B



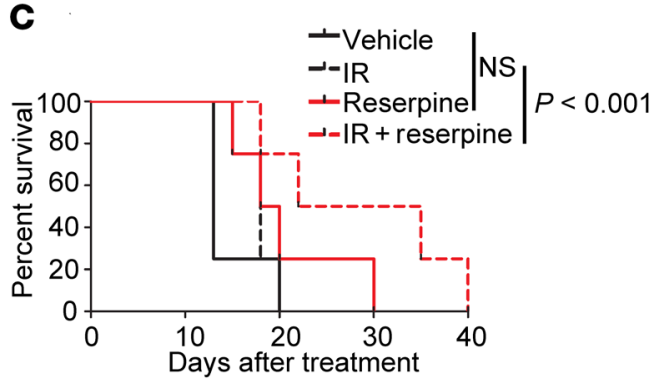

$\mathbf{F}$
D

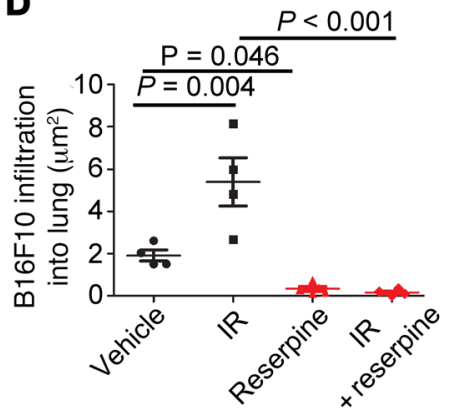

E


inoculation,

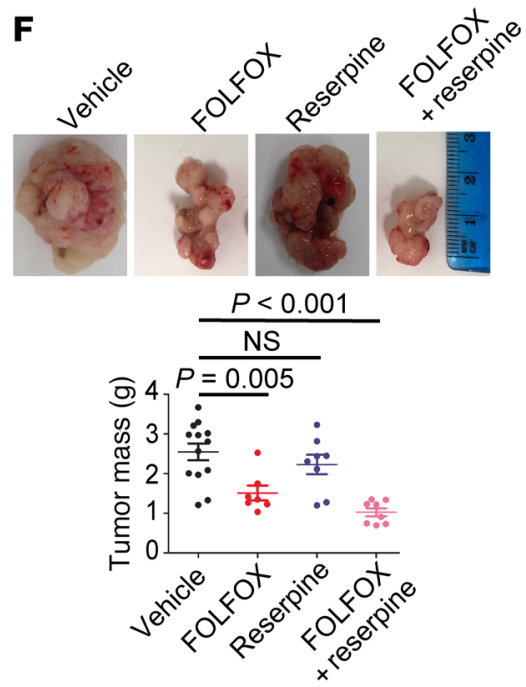

G

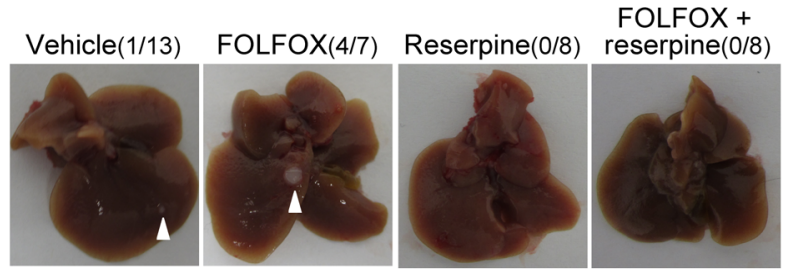

FOLFOX +

reserpine $(0 / 8)$

Figure 9. Administration of reserpine improves the outcomes of radio-/chemotherapies. (A) ELISA analysis of CD63 levels in the plasma from mice bearing B16F10 tumors of similar volume exposed or not to ionizing radiation (IR, 12 Gy), reserpine $(1 \mathrm{mg} / \mathrm{kg}$ ), or both. $n=4$ for all groups. (B) Analysis of tumor volume in B16F10 tumors (inoculated s.c. at $1 \times 10^{5}$ cells/mouse) in WT mice treated with vehicle or reserpine $(1 \mathrm{mg} / \mathrm{kg})$ upon reaching 30 to $50 \mathrm{~mm} \mathrm{~m}^{3}$. Three days later, vehicle- and reserpine-treated mice with similar tumor volumes underwent 12-Gy irradiation and continued their assigned vehicle or reserpine treatments 3 times per week $(n=4)$. (C) Kaplan-Meier survival analysis of B16F10 tumor-bearing mice treated as described in panel B until tumors reached $2000 \mathrm{~mm}^{3}(n=4)$. (D) Quantification of total area of B16F10 metastatic load in lungs from mice described in panel B $(n=4)$. (E) Schematic of the experiments combining reserpine with FOLFOX treatment of orthotopically inoculated MC38 colon tumors. (F) Representative images and the mass of MC38 tumors from animals treated as in panel E. $n=5$ for each group. (C) Representative images of livers from MC38 tumor-bearing mice described in panel $\mathbf{E}$. Arrowheads show macroscopic metastatic lesions found in $8 \%$ of vehicle-treated animals, $57 \%$ of FOLFOX only-treated animals, and none of the animals that received reserpine (with or without FOLFOX). Data are shown as mean \pm SEM. Statistical analysis was carried out using 1-way ANOVA with Tukey's multiple-comparison test (A, D, and F), 2-way ANOVA with Tukey's multiple-comparison test (B), or log-rank (Mantel-Cox) test (C). NS, not significant. Experiments were performed independently at least 3 times.

servative regions ( $\sim 1.8 \mathrm{~kb}$ upstream of exon 1 and $\sim 0.5 \mathrm{~kb}$ downstream of exon 1). Targeting vector, homology arms, and the conditional knockout region were generated by PCR using BAC clones RP23392N3 and RP24-61K11 from the C57BL/6J library as the templates. In the targeting vector (Supplemental Figure 5B), the Neo cassette was flanked by the self-deletion anchor sites and diphtheria toxin $\mathrm{A}$ was used for negative selection. C57BL/6J ES cells were used for gene targeting. Other mouse strains were generated by intercrossing; male and female 6-to 8-week-old littermates were used in all experiments.

Cell culture. Human 293T and HCT116 cells and mouse B16F10 cells were purchased from ATCC. Mouse MC38 colon adenocarcinoma (from S. Ostrand-Rosenberg, University of Maryland, Baltimore, Maryland, USA), TRAMP-C2-luc prostate neuroendocrine tumor cells (from L. Languino, Thomas Jefferson University, Philadelphia, Pennsylvania, USA), and MH6499c4 pancreatic ductal adenocarcinoma (from B.
Stanger, University of Pennsylvania, Philadelphia, Pennsylvania, USA) were gifted. All cells were maintained at $37^{\circ} \mathrm{C}$ with $5 \% \mathrm{CO}_{2}$ in DMEM supplemented with $10 \%$ heat-inactivated fetal bovine serum (FBS), 100 $\mathrm{U} / \mathrm{mL}$ penicillin-streptomycin, and L-glutamine.

TEV isolation, characterization, and assessment of uptake and production were carried out as previously described $(10,26)$. Briefly, TEVs were collected from (extracellular vesicle-free) media (Gibco, 11965-084) supernatants by ultracentrifugation and additional purification was carried out using discontinuous iodixanol gradients. TEVs were spun over $10 \% / 20 \% / 30 \%$ iodixanol layers at 350,000 $g(52,000$ rpm with Beckman SW 55 Ti rotor), $4^{\circ} \mathrm{C}$, for 120 minutes. Then, 10 fractions of $260 \mu \mathrm{L}$ each were collected starting from the top of the tube, diluted with $1 \mathrm{~mL}$ PBS, and resedimented at 100,000 $\mathrm{g}$ and $4^{\circ} \mathrm{C}$ for 70 minutes (Beckman Optima Ultracentrifuge and TLA-100.2 rotor at 53,000 rpm). Pellets in each fraction were resuspended in PBS 
and characterized for density by measuring the weight of each fraction $(\mathrm{g} / \mathrm{mL}$, indicated in Supplemental Table 1). Fractions 1 and 2 (1.11-1.12 $\mathrm{g} / \mathrm{mL}$ ) were the only ones containing TEVs.

Quantitative real-time PCR. Total RNA from ECs or tumors was extracted using TRIzol reagent (Life Technologies, 15596018) and analyzed by quantitative real-time PCR using SYBR Green Master Mix (Applied Biosystems, 4367659). Primers of indicated genes are listed in Supplemental Table 2.

TEV and liposome uptake in vitro was examined in the WT and Ch25h/- ECs cultured with TEV-free media and pretreated with vehicle or reserpine (diluted as previously described in ref. 26), followed by either DiD-labeled liposomes (FormuMax, F60103F-DD, $1 \mu \mathrm{g} / \mathrm{mL}$ ) or TEVs that were either labeled with DiD as described previously (26) or derived from B16F10 cells stably expressing GFP. Uptake of DiD was monitored by flow cytometry as described previously (26). Uptake of Gfp mRNA was analyzed after total RNA was isolated from ECs using TRIzol reagent and chloroform. RNA concentration and purity were determined by using a NanoDrop spectrophotometer (Thermo Fisher Scientific). An Applied Biosystems High-Capacity RNA-to-cDNA Kit was used to make cDNA. The Gfp mRNA level was measured by quantitative real-time PCR.

For assessment of TEV production by tumor cells in vitro, $6 \times 10^{6}$ cells were plated in $15-\mathrm{cm}$ dishes. Upon attachment of all cells, the media were removed and replaced with fresh media that contained 10\% extracellular vesicle-free FBS. The cells were treated with vehicle (DMSO) or reserpine $(10 \mu \mathrm{M})$. After 2 days, the conditioned media were collected for extracellular vesicle isolation and the total number of cells were counted for each condition. Ten microliters of isolated extracellular vesicles was submitted for nanoparticle tracking analysis (NTA), and $10 \mu \mathrm{L}$ was used for protein concentration. The number of vesicles per microliter and the amount of protein per vesicle were calculated correspondingly.

For assessment of TEV absolute number in plasma from tumor-bearing mice undergoing chemotherapy, $100 \mu \mathrm{L}$ of plasma was harvested and TEVs were isolated with an exosome isolation kit (Invitrogen, 4485229). Pellets were resuspended in 50-80 $\mu \mathrm{L}$ PBS and samples were assessed by NTA.

Flow cytometric analysis of ICBT and otherimmunologicaltechniques. Volume of tumors measured by caliper was calculated as width $\times$ width $\times$ length $\times 0.5$. Tumor tissues were dissected and digested with $1 \mathrm{mg} /$ $\mathrm{mL}$ Collagenase D (Roche, 11088882001) with $100 \mu \mathrm{g} / \mathrm{mL}$ DNase I (Roche, 10104159001) in RPMI medium with 2\% FBS for 1 hour with continuous agitation at $37^{\circ} \mathrm{C}$. The digestion mixture was passed through a $70-\mu \mathrm{m}$ cell strainer to prepare a single-cell suspension and washed with PBS supplemented with 2 mM EDTA and 1\% FBS. Single cells were stained with cell-surface antibodies: anti-CD45-APC-Cy7 (BioLegend, catalog 103115), anti-CD31-PE-Cy7 (BioLegend, catalog 102417), and anti-PDGFR $\alpha$-APC (BioLegend, catalog 135907). Data were acquired using an LSRFortessa flow cytometer (BD Biosciences) and analyzed with FlowJo software (Tree Star).

Enzyme-linked immunosorbent assay-based kits from Boster Bio, LLC (for ANGPT1 and ANGPT2) or Cusabio Technology, LLC (for CD63) were used to analyze levels of respective proteins in serum, tumor homogenates, or supernatants of EC cultures according to the manufacturers' instructions.

For the immunofluorescence analyses, tumor tissues were harvested, embedded in frozen OCT, and cryosectioned into 7- $\mu \mathrm{m}$ sections using a Leica CM3050 S cryostat. Tumor sections were fixed in acetone, washed with PBS, and then blocked with PBS containing 5\% goat serum and $1 \%$ BSA. Then, the sections were incubated for 12 hours at $4^{\circ} \mathrm{C}$ with anti-CD31 primary antibody (BD Biosciences, catalog 553370) diluted 1:200 in PBS, and followed by washing 3 times with PBS. Next, samples were incubated at room temperature for 1 hour with goat anti-rabbit secondary antibody (Invitrogen, catalog A-11006 or A-11007) diluted 1:500 in PBS. ProLong Gold Antifade reagent with DAPI (Invitrogen, P36935) was added after washing. Immunofluorescence images were captured with an Olympus BX51 microscope. CD31 staining area, distance, and number of blood vessels were analyzed with Metaphor software (Molecular Devices).

Isolation, culture, and proliferation/migration analyses of primary lung ECs was carried out as previously described (63). For Western blotting analysis of TIE2 phosphorylation, WT and Ch25h/ECs were isolated from respective mice and treated with PBS or MC38-derived vesicles $(20 \mu \mathrm{g} / \mathrm{mL})$ for 12 hours. Western blotting was carried out using a rabbit anti-mouse TIE2 polyclonal antibody (Thermo Fisher Scientific, catalog PA5-80103) and p-TIE2 was detected with rabbit anti-p-TIE2 antibody (Cell Signaling Technology, catalog 4221S), as described previously (66).

Analysis of plasma membrane fluidity was carried out as previously described (67). ECs treated as indicated were suspended in PBS at 4 $\times 10^{5} / \mathrm{mL}$ and incubated with the fluorescent probe 1,6-diphenyl-1,3,5hexatriene (Sigma-Aldrich, D208000; $3 \mu \mathrm{M}$ ) at $37^{\circ} \mathrm{C}$ for 20 minutes and held at $25.0 \pm 0.5^{\circ} \mathrm{C}$ for intensity polarization measurements using a Tecan Infinite F200 Fluorescence Microplate Reader System ( $\lambda$ ex $=313$ $\mathrm{nm} ; \lambda \mathrm{em}=460 \mathrm{~nm})$. The degree of cell membrane polarization was calculated using $P=(F 1-F 2) /(F 1+F 2)$, in which $F 1$ and $F 2$ refer to the fluorescence intensity of vertically and horizontally polarized components, respectively, with excitation vertically polarized.

Tumorigenesis studies. For the syngeneic subcutaneous tumor model, B16F10, MC38, or MH6499c4 tumor cells were inoculated into the right flank of indicated C57BL/6J mice. For xenograft studies, human HCT116 cells $\left(5 \times 10^{6}\right)$ were injected into NSG mice (The Jackson Laboratory). Studies using the orthotopic colon tumor growth cancer model were carried out as previously described (68). Briefly, after anesthesia, a $1.5-\mathrm{cm}$ nick was made in the abdominal wall (around) and the cecum was exteriorized and kept moist using PBS. Twenty-five microliters of the MC38 cell suspension $\left(2 \times 10^{7} / \mathrm{mL}\right)$ was injected into the cecal wall using a $30-\mathrm{G}$ needle and the injection site was covered with a cotton swab for 3 minutes to monitor for leakage. The cecum was gently returned to the abdominal wall, and then the abdominal wall and skin were sutured carefully. For the orthotopic prostatic cancer model, $1 \times 10^{6}$ TRAMP-C2-luc/GFP cells were injected into the prostate of WT or Ch25h/- mice. Tumor volumes were tracked via detecting bioluminescence intensity weekly.

FITC-lectin perfusion. MC38 cells $\left(1 \times 10^{6}\right)$ were injected into the right flank of WT and Ch $25 h^{-/}$mice. Fourteen days after injection, mice were anesthetized, injected with FITC-conjugated Lycopersicon esculentum (tomato) lectin (FITC-lectin) (Thermo Fisher Scientific, L32478; $100 \mu \mathrm{g} / \mathrm{mouse}$, i.v.) and allowed to circulate for 10 minutes. After that, the chest was opened rapidly and the vasculature was perfused with $30 \mathrm{~mL}$ of $4 \%$ paraformaldehyde (PFA) for 5 minutes. Tumor tissues were harvested and stored in PFA overnight before being frozen in OCT. Cryosections of tumors were stained with anti-CD31 for the whole blood vessels and FITC-positive areas were calculated with Metaphor software. 
Combination therapies. Reserpine (Sigma-Aldrich, 83580) was administered as previously described (26). Briefly, reserpine (dissolved in $0.1 \%$ ascorbic acid and diluted in $\left.\mathrm{ddH}_{2} \mathrm{O}\right)$ or vehicle $(0.1 \%$ ascorbic acid diluted in $\mathrm{ddH}_{2} \mathrm{O}$ ) was administered to $\mathrm{B} 16 \mathrm{~F} 10$ tumorbearing mice when the tumors reached $75 \mathrm{~mm}^{3}$ at the dose of $1 \mathrm{mg} /$ $\mathrm{kg}$ (i.p.; 3 times per week). Matched vehicle and reserpine mice with similar (100-130 $\mathrm{mm}^{3}$ ) tumor volumes were chosen to undergo irradiation using the Small Animal Radiation Research Platform (SARRP, Xstrahl Medical \& Life Sciences) 2 days later. Mice were anesthetized using inhaled $2.5 \%$ isoflurane and placed on the stage of the SARRP. Once the tumor isocenter was determined, delivery of the single 12-Gy dose was made using a $1 \times 1 \mathrm{~cm}$ collimated beam operating at $175 \mathrm{kV}, 15$ $\mathrm{mA}$, with copper filtration and the dose rate at $1.65 \mathrm{~Gy} / \mathrm{min}$. The beam was delivered at such an angle as to avoid the spine. Dosimetry was performed using EBT2 gafchromic films.

The ingredients for the FOLFOX regimen (oxaliplatin, PHR1528; 5-fluorouracil, F6627; folinic acid calcium salt hydrate, F7878 - all Sigma-Aldrich) were dissolved in PBS and administered $(150 \mathrm{mg} /$ $\mathrm{kg}$ folinic acid, $5 \mathrm{mg} / \mathrm{kg} 5$-fluorouracil, $1.4 \mathrm{mg} / \mathrm{kg}$ oxaliplatin; all i.p. every other day, with or without $1 \mathrm{mg} / \mathrm{kg}$ reserpine i.p.) into mice 10 days after the animals were inoculated with $5 \times 10^{5} \mathrm{MC} 38$ cells injected into the cecum. All mice were sacrificed 45 days after tumor inoculation and tumor, liver, and intestine were harvested for the histopathologic analysis. Sunitinib (BioVision, 1611) was dissolved in a vehicle (composed of carboxymethylcellulose sodium $[0.5 \% \mathrm{w} / \mathrm{v}], \mathrm{NaCl}[1.8 \% \mathrm{w} / \mathrm{v}]$, Tween $80[0.4 \% \mathrm{w} / \mathrm{v}]$, and benzyl alcohol $[0.9 \% \mathrm{w} / \mathrm{v}]$ in water; the whole formulation was adjusted to $\mathrm{pH}$ 6.0). Rebastinib was dissolved in $0.4 \%$ hydroxypropyl methylcellulose. MC 38 or B16F10 cells $\left(5 \times 10^{5}\right)$ were inoculated into the right flank of WT mice. Nine days after tumor inoculation, mice were treated with sunitinib (40 mg/ $\mathrm{kg}$, gavage) 3 times per week with or without reserpine $(1 \mathrm{mg} / \mathrm{kg}$, i.p.) every other day or rebastinib (20 mg/kg, gavage) twice per week.

RNA sequencing. Primary lung ECs from $\mathrm{Ch} 25 \mathrm{~h}^{-/-}$mice were pretreated with vehicle or reserpine (10 $\mu \mathrm{M}$ for 8 hours) followed by treatment with MC38 TEVs $(20 \mu \mathrm{g} / \mathrm{mL})$ or PBS for 12 hours in vitro and total RNA was isolated using the RNeasy Plus Mini Kit (QIAGEN) and analyzed for Angpt 2 mRNA levels by qPCR. These samples were then used for RNA sequencing (carried out as previously described, ref. 69). Raw reads were mapped to the mouse reference transcriptome (Ensembl) using Kallisto version 0.46.0. Raw data are available in the NCBI's Gene Expression Omnibus database (GEO GSE163941). All subsequent analyses were carried out using the statistical computing environment $\mathrm{R}$ version 4.0.0 in RStudio and Bioconductor version 3.11.1 as described in the supplemental material.

Statistics. All experiments described here are representative of at least 3 independent experiments $(n>5$ mice for each group unless otherwise specified). For in vitro experiments, cells or tissues from each animal were incorporated in triplicate. All data are shown as mean \pm SEM. Statistical analyses were conducted using GraphPad Prism 7 software. Comparisons between 2 groups were conducted with a 2-tailed Student's $t$ test and multiple comparisons were performed using 1-way ANOVA or 1-way ANOVA followed by Bonferroni's post hoc test. Tumor growth curve analysis was conducted with repeatedmeasures 2-way ANOVA (mixed model) followed by Bonferroni's post hoc test. Kaplan-Meier curves were used to analyze the survival data, and Cox regression was used to compute hazard ratios. $P$ values of less than 0.05 were considered significant.

Study approval. Use of preexisting human archival decodified and deidentified CRC tissue arrays, previously collected under informed consent, and samples that could not be directly or indirectly linked to individual human subjects was exempt from institutional review or approved by the IRB of the Medical College of Wisconsin.

All animal experiments were approved by the Institutional Animal Care and Use Committee of the University of Pennsylvania and were carried out in accordance with the IACUC guidelines. All mice had water ad libitum and were fed regular chow. Mice were maintained in a specific pathogen-free facility in accordance with American Association for Laboratory Animal Science guidelines. Littermates from different cages were randomly assigned to the experimental groups. These randomized experimental cohorts were either cohoused or systematically exposed to the bedding of other groups to ensure equal exposure to the microbiota of all groups.

\section{Author contributions}

SYF, ZL, HR, JAD, CK, and SWR designed the research. ZL, AO, IIV, ARP, FZ, CC, PY, RMD, HZ, RK, YS, ER, ATY, EK, and DPB performed the experiments and interpreted the data. SYF, ZL, HR, JAD, $\mathrm{CK}$, and SWR wrote the manuscript with the help of all authors.

\section{Acknowledgments}

This work was supported by NIH/NCI grants R01 CA247803 (to SYF and DPB), R01 CA240814 (to SYF and HR), and P01 CA165997 (to JAD, CK, and SYF). We are thankful for support for RMD and equipment for characterizing TEVs from The Extracellular Vesicle Core Facility, School of Veterinary Medicine, University of Pennsylvania. We are grateful to Elena Voronov (Ben Gurion University of the Negev, Beer Sheva, Israel) and Bang-Jin Kim (SWR lab) for valuable advice. We thank Susan OstrandRosenberg (University of Maryland, Baltimore, Maryland, USA), Ben Stanger (University of Pennsylvania), and Lucia Languino (Thomas Jefferson University) for reagents. We also thank Ze'ev Ronai (Sanford Burnham Prebys Medical Discovery Institute, San Diego, California, USA), Gennady Belitsky (N.N. Blokhin National Medical Research Center of Oncology, Moscow, Russia), Ana Gamero (Temple University, Philadelphia, Pennsylvania, USA), Igor Astsaturov (Fox Chase Cancer Center, Philadelphia, Pennsylvania, USA), and the members of the Fuchs, Koumenis, and Ryeom labs for critical suggestions.

Address correspondence to: Serge Y. Fuchs, Dept. of Biomedical Sciences, School of Veterinary Medicine, University of Pennsylvania, 380 S. University Ave, Hill 316, Philadelphia, Pennsylvania 19104, USA. Phone: 1.215.573.6949; Email: syfuchs@upenn.edu.
1. Mittelbrunn M, Sanchez-Madrid F. Intercellular communication: diverse structures for exchange of genetic information. Nat Rev Mol Cell Biol. 2012;13(5):328-335.
2. Murk JL, et al. The plasticity of multivesicular bodies and the regulation of antigen presentation. Semin Cell Dev Biol. 2002;13(4):303-311.

3. Thery C, et al. Exosomes: composition, biogenesis and function. Nat Rev Immunol. 2002;2(8):569-579.

4. Todorova D, et al. Extracellular vesicles in angiogenesis. Circ Res. 2017;120(10):1658-1673. 
5. Becker A, et al. Extracellular vesicles in cancer: cell-to-cell mediators of metastasis. Cancer Cell. 2016;30(6):836-848.

6. Choi D, et al. Extracellular vesicle communication pathways as regulatory targets of oncogenic transformation. Semin Cell Dev Biol. 2017;67:11-22.

7. Kalluri R. The biology and function of exosomes in cancer. J Clin Invest. 2016;126(4):1208-1215.

8. De Palma M, et al. Microenvironmental regulation of tumour angiogenesis. Nat Rev Cancer. 2017;17(8):457-474

9. Rechavi O, et al. Intercellular exchange of proteins: the immunecell habit of sharing. FEBS Lett. 2009;583(11):1792-1799.

10. Peinado $\mathrm{H}$, et al. Melanoma exosomes educate bone marrow progenitor cells toward a prometastatic phenotype through MET. Nat Med. 2012;18(6):883-891.

11. Lobb RJ, et al. Exosomes: key mediators of metastasis and pre-metastatic niche formation. Semin Cell Dev Biol. 2017;67:3-10.

12. Aleckovic M, Kang Y. Regulation of cancer metastasis by cell-free miRNAs. Biochim Biophys Acta. 2015;1855(1):24-42.

13. Streubel B, et al. Lymphoma-specific genetic aberrations in microvascular endothelial cells in B-cell lymphomas. $N$ Engl J Med 2004;351(3):250-259.

14. Ehnfors J, et al. Horizontal transfer of tumor DNA to endothelial cells in vivo. Cell Death Differ. 2009;16(5):749-757.

15. Nazarenko I, et al. Cell surface tetraspanin Tspan 8 contributes to molecular pathways of exosome-induced endothelial cell activation. Cancer Res. 2010;70(4):1668-1678.

16. Aslan C, et al. Tumor-derived exosomes: implication in angiogenesis and antiangiogenesis cancer therapy. J Cell Physiol. 2019;234(10):16885-16903.

17. Namee NM, O’Driscoll L. Extracellular vesicles and anti-cancer drug resistance. Biochim Biophys Acta Rev Cancer. 2018;1870(2):123-136.

18. Ni J, et al. Exosomes in cancer radioresistance. Front Oncol. 2019;9:869.

19. O'Neill CP, et al. Role of extracellular vesicles (EVs) in cell stress response and resistance to cancer therapy. Cancers (Basel). 2019;11(2):E136.

20. Bandari SK, et al. Chemotherapy induces secretion of exosomes loaded with heparanase that degrades extracellular matrix and impacts tumor and host cell behavior. Matrix Biol. 2018;65:104-118.

21. Blyth BJ, et al. Radiation therapy-induced metastasis: radiobiology and clinical implications. Clin Exp Metastasis. 2018;35(4):223-236.

22. Kaplan HS, Murphy ED. The effect of local roentgen irradiation on the biological behavior of a transplantable mouse carcinoma; increased frequency of pulmonary metastasis. J Natl Cancer Inst. 1949;9(5-6):407-413.

23. Karagiannis GS, et al. Chemotherapy-induced metastasis: mechanisms and translational opportunities. Clin Exp Metastasis. 2018;35(4):269-284.

24. Keklikoglou I, et al. Chemotherapy elicits prometastatic extracellular vesicles in breast cancer models. Nat Cell Biol. 2019;21(2):190-202.

25. Mutschelknaus L, et al. Radiation alters the cargo of exosomes released from squamous head and neck cancer cells to promote migration of recipient cells. Sci Rep. 2017;7(1):12423.
26. Ortiz A, et al. An interferon-driven oxysterolbased defense against tumor-derived extracellular vesicles. Cancer Cell. 2019;35(1):33-45.e6.

27. Gui J, et al. Activation of $\mathrm{p} 38 \alpha$ stress-activated protein kinase drives the formation of the pre-metastatic niche in the lungs. Nat Cancer. 2020;1(6):603-619.

28. Katlinskaya YV, et al. Suppression of type I interferon signaling overcomes oncogene-induced senescence and mediates melanoma development and progression. Cell Rep. 2016;15(1):171 180.

29. Anggakusuma, et al. Interferon-inducible cholesterol-25-hydroxylase restricts hepatitis $\mathrm{C}$ virus replication through blockage of membranous web formation. Hepatology. 2015;62(3):702-714.

30. Liu SY, et al. Interferon-inducible cholesterol25-hydroxylase broadly inhibits viral entry by production of 25-hydroxycholesterol. Immunity. 2013;38(1):92-105.

31. Blanc M, et al. The transcription factor STAT-1 couples macrophage synthesis of 25-hydroxycholesterol to the interferon antiviral response. Immunity. 2013;38(1):106-118.

32. Luu B, Moog C. Oxysterols: biological activities and physicochemical studies. Biochimie. 1991;73(10):1317-1320.

33. Bhattacharya $\mathrm{S}$, et al. Triggering ubiquitination of IFNAR1 protects tissues from inflammatory injury. EMBO Mol Med. 2014;6(3):384-397.

34. Tait CR, Jones PF. Angiopoietins in tumours: the angiogenic switch. J Pathol. 2004;204(1):1-10.

35. Bergers G, Hanahan D. Modes of resistance to anti-angiogenic therapy. Nat Rev Cancer. 2008;8(8):592-603.

36. De Palma M, Naldini L. Angiopoietin-2 TIEs up macrophages in tumor angiogenesis. Clin Cancer Res. 2011;17(16):5226-5232.

37. Payne S, et al. Endothelial-specific cre mouse models. Arterioscler Thromb Vasc Biol. 2018;38(11):2550-2561

38. Daly C, et al. Angiopoietin-2 functions as a Tie2 agonist in tumor models, where it limits the effects of VEGF inhibition. Cancer Res. 2013;73(1):108-118.

39. Rigamonti $\mathrm{N}$, et al. Role of angiopoietin- 2 in adaptive tumor resistance to VEGF signaling blockade. Cell Rep. 2014;8(3):696-706

40. Harney AS, et al. The selective Tie 2 inhibitor rebastinib blocks recruitment and function of Tie2(Hi) macrophages in breast cancer and pancreatic neuroendocrine tumors. Mol Cancer Ther. 2017;16(11):2486-2501.

41. Schmittnaegel M, et al. Dual angiopoietin-2 and VEGFA inhibition elicits antitumor immunity that is enhanced by PD-1 checkpoint blockade. Sci Transl Med. 2017;9(385):eaak9670.

42. Yaffe D, et al. The ins and outs of vesicular monoamine transporters. J Gen Physiol. 2018;150(5):671-682

43. Kowal J, et al. Biogenesis and secretion of exosomes. Curr Opin Cell Biol. 2014;29:116-125.

44. Mathieu M, et al. Specificities of secretion and uptake of exosomes and other extracellular vesicles for cell-to-cell communication. Nat Cell Biol. 2019;21(1):9-17.

45. Wendler F, et al. Extracellular vesicles swarm the cancer microenvironment: from tumor-stroma communication to drug intervention. Oncogene. 2017;36(7):877-884.

46. Hochster HS, et al. Safety and efficacy of oxaliplatin and fluoropyrimidine regimens with or without bevacizumab as first-line treatment of metastatic colorectal cancer: results of the TREE Study. J Clin Oncol. 2008;26(21):3523-3529.

47. Sasaki S, et al. Involvement of prokineticin 2-expressing neutrophil infiltration in 5-fluorouracil-induced aggravation of breast cancer metastasis to lung. Mol Cancer Ther. 2018;17(7):1515-1525.

48. Chang J, et al. Chemotherapy-generated cell debris stimulates colon carcinoma tumor growth via osteopontin. FASEB J. 2019;33(1):114-125.

49. Bhattacharya S, et al. Anti-tumorigenic effects of type 1 interferon are subdued by integrated stress responses. Oncogene. 2013;32(36):4214-4221.

50. Huangfu WC, et al. Inflammatory signaling compromises cell responses to interferon alpha. Oncogene. 2012;31(2):161-172.

51. Katlinski KV, et al. Inactivation of interferon receptor promotes the establishment of immune privileged tumor microenvironment. Cancer Cell. 2017;31(2):194-207.

52. Zheng $\mathrm{H}$, et al. Vascular endothelial growth factor-induced elimination of the type 1 interferon receptor is required for efficient angiogenesis. Blood. 2011;118(14):4003-4006.

53. Zhu D, et al. The ROS-mediated activation of STAT-3/VEGF signaling is involved in the 27-hydroxycholesterol-induced angiogenesis in human breast cancer cells. Toxicol Lett. 2016;264:79-86.

54. Cox DC, et al. Effects of cholesterol and 25-hydroxycholesterol on smooth muscle cell and endothelial cell growth. Lipids. 1988;23(2):85-88.

55. Horie K, et al. Exosomes expressing carbonic anhydrase 9 promote angiogenesis. Biochem Biophys Res Commun. 2017;492(3):356-361.

56. Huang Z, Feng Y. Exosomes derived from hypoxic colorectal cancer cells promote angiogenesis through Wnt4-induced $\beta$-catenin signaling in endothelial cells. Oncol Res. 2017;25(5):651-661.

57. Kucharzewska P, et al. Exosomes reflect the hypoxic status of glioma cells and mediate hypoxia-dependent activation of vascular cell during tumor development. Proc Natl Acad Sci U S A. 2013;110(18):7312-7317.

58. Li CC, et al. Glioma microvesicles carry selectively packaged coding and non-coding RNAs which alter gene expression in recipient cells. RNA Biol. 2013;10(8):1333-1344.

59. Treps L, et al. Glioblastoma stem-like cells secrete the pro-angiogenic VEGF-A factor in extracellular vesicles. J Extracell Vesicles. 2017;6(1):1359479.

60. Sato S, et al. EPHB2 carried on small extracellular vesicles induces tumor angiogenesis via activation of ephrin reverse signaling. JCI Insight. 2019;4(23):132447.

61. Mathiyalagan P, et al. Angiogenic mechanisms of human $\mathrm{CD} 4^{+}$stem cell exosomes in the repair of ischemic hindlimb. Circ Res. 2017;120(9):1466-1476.

62. Qu L, et al. Exosome-transmitted lncARSR 


\section{RESEARCH ARTICLE}

The Journal of Clinical Investigation

promotes sunitinib resistance in renal cancer by acting as a competing endogenous RNA. Cancer Cell. 2016;29(5):653-668.

63. Minami T, et al. The calcineurin-NFAT-angiopoietin-2 signaling axis in lung endothelium is critical for the establishment of lung metastases. Cell Rep. 2013;4(4):709-723.

64. Peck AR, et al. Loss of nuclear localized and tyrosine phosphorylated Stat 5 in breast cancer predicts poor clinical outcome and increased risk of antiestrogen therapy failure. J Clin Oncol. 2011;29(18):2448-2458.

65. Alva JA, et al. VE-Cadherin-Cre-recombinase transgenic mouse: a tool for lineage analysis and gene deletion in endothelial cells. Dev Dyn. 2006;235(3):759-767.

66. Fuchs SY, Ronai Z. Ubiquitination and degradation of ATF2 are dimerization dependent. $\mathrm{Mol}$ Cell Biol. 1999;19(5):3289-3298.

67. Shinitzky M, Inbar M. Difference in microviscos- ity induced by different cholesterol levels in the surface membrane lipid layer of normal lymphocytes and malignant lymphoma cells. J Mol Biol. 1974;85(4):603-615.

68. Tseng W, et al. Orthotopic mouse model of colorectal cancer. JVis Exp. 2007(10):484.

69. Amorim CF, et al. Variable gene expression and parasite load predict treatment outcome in cutaneous leishmaniasis. Sci Transl Med. 2019;11(519):eaax4204. 